\title{
Inverse problems for Jacobi operators IV: Interior mass-spring perturbations of semi-infinite systems*
}

\author{
Rafael del Rio \\ Departamento de Física Matemática \\ Instituto de Investigaciones en Matemáticas Aplicadas y en Sistemas \\ Universidad Nacional Autónoma de México \\ C.P. 04510, México D.F. \\ delrio@iimas.unam.mx \\ Mikhail Kudryavtsev \\ Department of Mathematics \\ Institute for Low Temperature Physics and Engineering \\ Lenin Av. 47, 61103 \\ Kharkov, Ukraine \\ kudryavtsev@onet.com.ua \\ Luis O. Silva \\ Departamento de Física Matemática \\ Instituto de Investigaciones en Matemáticas Aplicadas y en Sistemas \\ Universidad Nacional Autónoma de México \\ C.P. 04510, México D.F. \\ silva@iimas.unam.mx
}

\begin{abstract}
This work gives results on the interplay of the spectra of two Jacobi operators corresponding to an infinite mass-spring system and a modification of it obtained by changing one mass and one spring of the system. It is shown that the system can be recovered from these two spectra. Necessary and sufficient conditions for two sequences to be the spectra of the mass-spring system and the perturbed one are provided.
\end{abstract}

Mathematics Subject Classification(2010): 34K29, 47A75, 47B36, 70F17,

Keywords: Infinite mass-spring system; Jacobi matrices; Two-spectra inverse problem.

*Research partially supported by UNAM-DGAPA-PAPIIT IN105414 


\section{Introduction}

Inverse spectral problems are concerned with the quest of information determining an operator from its spectral data. These problems have various applications in physics and other sciences. Usually, we do not possess all the information that defines the operator modeling a certain physical system, however it is possible to measure physical quantities related to the spectrum of the operator and use these data to gain some information about the operator, thence about the system.

The kind of inverse spectral problem studied in the present work is the so called two spectra inverse problem in which one is given the spectra of an operator and a perturbation of it with the goal of recovering the operator from these two spectra.

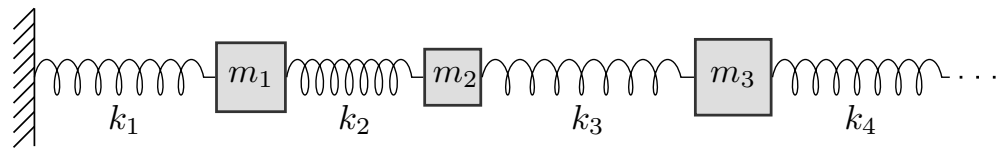

Figure 1: Semi-infinite mass-spring system

Consider the linear semi-infinite mass spring system illustrated in Fig. 1. This mechanical system, with masses $\left\{m_{j}\right\}_{j=1}^{\infty}$ and spring constants $\left\{k_{j}\right\}_{j=1}^{\infty}$, is modeled by a Jacobi operator $J$ associated with the Jacobi matrix

$$
\left(\begin{array}{ccccc}
q_{1} & b_{1} & 0 & 0 & \cdots \\
b_{1} & q_{2} & b_{2} & 0 & \cdots \\
0 & b_{2} & q_{3} & b_{3} & \\
0 & 0 & b_{3} & q_{4} & \ddots \\
\vdots & \vdots & & \ddots & \ddots
\end{array}\right) .
$$

where

$$
q_{j}=-\frac{k_{j+1}+k_{j}}{m_{j}}, \quad b_{j}=\frac{k_{j+1}}{\sqrt{m_{j} m_{j+1}}}, \quad j \in \mathbb{N} .
$$

In solid state physics, the mass-spring system of Fig. 1 is used as a model of one-dimensional infinite harmonic crystals (see [38, p. 22]). A finite mass-spring system can be used to study molecular vibrations, where the chemical bounds between atoms (masses) are modeled by springs [34].

Assuming that the movement of the system takes place within the regime of validity of the Hooke law, one derives a Jacobi operator with entries given by (1.2) from the dynamics equations (cf. [14, 24] for the finite case). If the

spectrum of $J$ is discrete, the movement of the system is the superposition of 
harmonic oscillations whose frequencies are the square root of the eigenvalues' absolute values.

In our two spectra inverse problem, one wants to find the matrix entries corresponding to operator $J$ from the spectra of $J$ and a perturbation of it. The perturbed operator, denoted $\widetilde{J}_{n}$, has (2.5) as its matrix representation and corresponds to the linear semi-infinite mass-spring system given in Fig. 2 .

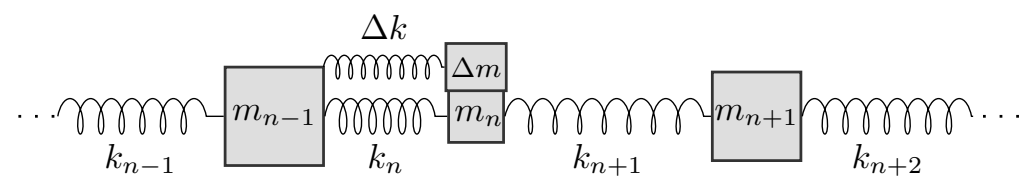

Figure 2: Perturbed semi-infinite mass-spring system $(n \geq 2)$

Inverse spectral problems for Jacobi operators have been amply studied (see for instance [6, 7, 8, 13, 15, 18, 19, 20, 25, 26, 28] for the finite case and [9, 12, 13, 16, 17, 32, 33] for the infinite case). However, inverse spectral problems that involve the kind of perturbation producing $\widetilde{J}_{n}$ from $J$ have been treated, to the best of our knowledge, only in the finite case [8, 25, 26]. Yet, this sort of perturbation arises in a natural way from the view point of physics: it corresponds to the modification of one mass and spring constant at any place in the chain. Noteworthily, by solving our inverse problem, we recover the masses and spring constants of the system and the parameters of the perturbation from the knowledge of the natural frequencies of vibration of the original system and the perturbed one. In particular, in the finite case, solving the inverse problem allows measuring micromasses with the help of microcantilevers [35, 36].

To tackle the inverse problem, we use the characterization of the relative distribution of the spectra of $J$ and $\widetilde{J}_{n}$ given in [11]. Here a central role is played by the Green functions of the original and perturbed operators. The Green function is the diagonal entry of the matrix of the resolvent operator at the point corresponding to the place in the chain where the perturbation occurs. Since the Green functions are fundamental for our direct and inverse spectral analysis, we give necessary and sufficient conditions for a meromorphic Herglotz function to be a Green function of a Jacobi operator with discrete spectrum (Proposition 3.7). Direct spectral analysis of the operator and its perturbation gives a point on the real line which is determined by the perturbation parameters and seems to act as an "attractor" for the eigenvalues as they are perturbed. This is relevant for choosing proper enumerations of the set of eigenvalues. An important conclusion of the spectral analysis is Theorem 4.9 on the convergence of the sum of the difference of eigenvalues (cf. [21]).

Having solved the direct spectral problems, we turn to solving the conditional inverse problem. We determine the input spectral data needed for the 
reconstruction of the Green function (Proposition 5.4). Moreover, in Theorems 5.6 and 5.7, we characterize the set of Jacobi operators that share the same Green function and the solutions of the two spectra inverse problem. An important ingredient for this result is Proposition 3.3 which is the key to the theory of interior perturbations developed in [13. Finally, we give necessary and sufficient conditions for two sequences to be the spectra of a Jacobi operator $J$ and its perturbation $\widetilde{J}_{n}$ (Theorems 5.9 and 5.10).

This paper is a continuation of recent work on the matter [8, 9, 10, 11] and presents substantial generalizations of previous results. We are now able to manage the situation where the perturbation takes place at any arbitrary interior mass and spring of the system. In the course of obtaining these generalizations, unexpected nuances appeared, so it was necessary to recur to results not needed before and develop new techniques. It is remarkable that in the solution of the concrete problem we have posed, various crucial problems of modern analysis converge: the moment problem, the subtle problem of density of polynomials in $L_{2}$ spaces, and various aspects in the theory of functions.

The paper is organized as follows. In the next section, we introduce the Jacobi operators and the finite-rank perturbation performed on them. Some preparatory facts on Jacobi operators and their Weyl $m$-functions are accounted for in this section. In Section 3, the Green functions are defined and a crucial formula is brought in. Here we state the necessary and sufficient conditions for a meromorphic Herglotz function to be the Green function of an operator with discrete spectrum. In Section 4, the key formula (4.2) is considered and results are given which describe how the eigenvalues of the perturbed operator depend on the perturbation parameters. Section 5 provides necessary and sufficient conditions on two sequences of points to be eigenvalues of an operator $J$ and a perturbation of it. Finally, in the Appendix, we include a result on the representation of Weyl $m$-functions of Jacobi operators on the basis of a classical result due to M. G. Krein.

\section{Jacobi operators}

For a sequence $f=\left\{f_{k}\right\}_{k=1}^{\infty}$ of complex numbers, consider the second order difference expressions

$$
\begin{aligned}
& (\Upsilon f)_{k}:=b_{k-1} f_{k-1}+q_{k} f_{k}+b_{k} f_{k+1} \quad k \in \mathbb{N} \backslash\{1\}, \\
& (\Upsilon f)_{1}:=q_{1} f_{1}+b_{1} f_{2}
\end{aligned}
$$

where $q_{k} \in \mathbb{R}$ and $b_{k}>0$ for any $k \in \mathbb{N}$. We remark that (2.1b) can be seen as a boundary condition.

Let $l_{2}(\mathbb{N})$ be the space of square summable complex sequences. In this 
Hilbert space, define the operator $J_{0}$ whose domain contains only the sequences having a finite number of non-zero elements and is given by $J_{0} f:=\Upsilon f$. Clearly, the operator $J_{0}$ is symmetric and therefore closable, so one can consider the operator $\overline{J_{0}}$ being its closure. It turns out that $\overline{J_{0}}$ is the operator whose matrix representation with respect to the canonical basis $\left\{\delta_{n}\right\}_{n=1}^{\infty}$ in $l_{2}(\mathbb{N})$ is

$$
\left(\begin{array}{ccccc}
q_{1} & b_{1} & 0 & 0 & \cdots \\
b_{1} & q_{2} & b_{2} & 0 & \cdots \\
0 & b_{2} & q_{3} & b_{3} & \\
0 & 0 & b_{3} & q_{4} & \ddots \\
\vdots & \vdots & & \ddots & \ddots
\end{array}\right)
$$

See [2, Sec. 47] for the definition of the matrix representation of an unbounded symmetric operator.

One of the following two possibilities for the deficiency indices of $\overline{J_{0}}$ holds [1, Chap. 4, Sec. 1.2]:

$$
\begin{aligned}
& n_{+}\left(\overline{J_{0}}\right)=n_{-}\left(\overline{J_{0}}\right)=1, \\
& n_{+}\left(\overline{J_{0}}\right)=n_{-}\left(\overline{J_{0}}\right)=0 .
\end{aligned}
$$

Let $J$ be a self-adjoint extension of $J_{0}$. Thus, in view of (2.3), the von Neumann extension theory tells us that either $J$ is a proper closed symmetric extension of $\overline{J_{0}}$ or $J=\overline{J_{0}}$. In the general case, there are various operators $J$ associated with the matrix (2.2) and we referred to them generically as Jacobi operators associated with (2.2).

Within the regime of validity of the Hooke law, the Jacobi operator $J$ models the semi-infinite linear mass-spring system of Fig. 1 [9, 11] with (1.2). See [14, 24] for an explanation of the deduction of these formulae in the finite case.

Fix $n \in \mathbb{N}$ and consider, along with the self-adjoint operator $J$, the operator

$$
\begin{aligned}
\widetilde{J}_{n}=J & +\left[q_{n}\left(\theta^{2}-1\right)+\theta^{2} h\right]\left\langle\delta_{n}, \cdot\right\rangle \delta_{n} \\
& +b_{n}(\theta-1)\left(\left\langle\delta_{n}, \cdot\right\rangle \delta_{n+1}+\left\langle\delta_{n+1}, \cdot\right\rangle \delta_{n}\right) \\
& +b_{n-1}(\theta-1)\left(\left\langle\delta_{n-1}, \cdot\right\rangle \delta_{n}+\left\langle\delta_{n}, \cdot\right\rangle \delta_{n-1}\right), \quad \theta>0, \quad h \in \mathbb{R},
\end{aligned}
$$

where it has been assumed that $b_{0}=0$. Clearly, $\widetilde{J}_{n}$ is a self-adjoint extension of the operator whose matrix representation with respect to the canonical basis in $l_{2}(\mathbb{N})$ is a Jacobi matrix obtained from (2.2) by modifying the entries $b_{n-1}, q_{n}, b_{n}$. For instance, if $n>2, \widetilde{J}_{n}$ is a self-adjoint extension (possibly not proper) of the 
operator whose matrix representation is

$$
\left(\begin{array}{ccccccc}
q_{1} & b_{1} & 0 & 0 & 0 & 0 & \cdots \\
b_{1} & \ddots & \ddots & 0 & 0 & 0 & \cdots \\
0 & \ddots & q_{n-1} & \theta b_{n-1} & 0 & 0 & \cdots \\
0 & 0 & \theta b_{n-1} & \theta^{2}\left(q_{n}+h\right) & \theta b_{n} & 0 & \cdots \\
0 & 0 & 0 & \theta b_{n} & q_{n+1} & \theta b_{n+1} & \\
0 & 0 & 0 & 0 & b_{n+1} & q_{n+2} & \ddots \\
\vdots & \vdots & \vdots & \vdots & & \ddots & \ddots
\end{array}\right) .
$$

Note that $\widetilde{J}_{n}$ is obtained from $J$ by a rank-three perturbation when $n>1$, and a rank-two perturbation otherwise.

The operator $\widetilde{J}_{n}$ serves as a model of the perturbed mass spring system of Fig. 2, where

$$
\Delta m=m_{n}\left(\theta^{-2}-1\right) \quad \text { and } \quad \Delta k=-h m_{n} .
$$

By setting $f_{1}=1$, a solution of the equations

$$
\begin{aligned}
& (\Upsilon f)_{1}:=z f_{1}, \\
& (\Upsilon f)_{k}:=z f_{k}, \quad k \in \mathbb{N} \backslash\{1\},
\end{aligned}
$$

can be found uniquely by recurrence. This solution, denoted by $\pi(z)=\left\{\pi_{k}(z)\right\}$, is such that $\pi_{k}(z)$ is a polynomial of degree $k-1$. Alongside this sequence, define the sequence $\xi(z)$ as the solution of $(\underline{2.7 \mathrm{~b}})$ after setting $f_{1}=0$ and $f_{2}=b_{1}^{-1}$. Thus, $\xi_{k}(z)$ is a polynomial of degree $k-2$. The elements of the sequence $\pi(z)$, respectively $\xi(z)$, are referred to as the polynomials of the first, respectively second, kind associated with the matrix (2.2). By comparing (2.1) with (2.7), one concludes that for $\pi(z)$ to be in $\operatorname{ker}\left(J_{0}^{*}-z I\right)$, it is necessary and sufficient that $\pi(z)$ be an element of $l_{2}(\mathbb{N})$. Of course, $\pi(z) \in \operatorname{ker}(J-z I)$, if and only if $\pi(z) \in \operatorname{dom}(J)$.

It follows from the definition of the operator $J$ that

$$
\delta_{k}=\pi_{k}(J) \delta_{1} \quad \forall k \in \mathbb{N} .
$$

This implies that $J$ is simple and $\delta_{1}$ is a cyclic vector (see [2, Sec. 69]). Therefore, by defining

$$
\rho(t):=\left\langle\delta_{1}, E(t) \delta_{1}\right\rangle, \quad t \in \mathbb{R},
$$

where $E$ is the resolution of the identity given by the spectral theorem, one has, due to [2, Sec. 69, Thm. 2]), that there is a unitary map $\Phi: L_{2}(\mathbb{R}, \rho) \rightarrow l_{2}(\mathbb{N})$ such that $\Phi^{-1} J \Phi$ is the multiplication by the independent variable defined in its maximal domain. We call the function given by (2.9) the spectral function 
of the Jacobi operator J. Moreover, due to [2, Sec. 69, Thm. 2]), it follows from (2.8) that the function $\pi_{k} \Upsilon_{\mathbb{R}}$ belongs to $L_{2}(\mathbb{R}, \rho)$ for all $k \in \mathbb{N}$, i. e., all moments of $\rho$ exists (see also [1, Thm.4.1.3]). The equation (2.8) means that

$$
\Phi \pi_{k}=\delta_{k}, \quad \forall k \in \mathbb{N}
$$

which implies that the polynomials are dense in $L_{2}(\mathbb{R}, \rho)$ since $\Phi$ is unitary. Thus, the discussion above has proven one direction of the following assertion which follows directly from [1, Thms. 2.3.3 and 4.1.4].

Theorem 2.1. A function $\rho$ is the spectral function of a Jacobi operator if and only if $\int_{\mathbb{R}} d \rho=1$, all the polynomials are in $L_{2}(\mathbb{R}, \rho)$ and they are dense in this space.

Remark 2.2. Recall that $J$ is either a proper self-adjoint restriction of $J_{0}^{*}$ or $J=\overline{J_{0}}$ depending on the alternative given in (2.3). Thus, for any measure $\rho$ such that all polynomials are a dense linear subset of $L_{2}(\mathbb{R}, \rho)$, there exists a Jacobi operator $J$, viz. a canonical self-adjoint extension of the operator whose matrix representation is a Jacobi matrix, such that $\rho$ and $J$ are related by (2.9).

Remark 2.3. Any measure with finite support is the spectral measure of the operator associated with some finite Jacobi matrix.

Definition 2.4. The Weyl $m$-function is defined as follows

$$
m(z):=\left\langle\delta_{1},(J-z I)^{-1} \delta_{1}\right\rangle, \quad z \notin \sigma(J),
$$

where $\sigma(J)$ denotes the spectrum of $J$.

By the map $\Phi$, it immediately follows from this definition that

$$
m(z)=\int_{\mathbb{R}} \frac{d \rho(t)}{t-z} .
$$

Thus, by the Nevanlinna representation theorem (see [30, Thm.5.3]), $m(z)$ is a Herglotz function. Recall that a Herglotz function $f$ (also called Pick or Nevanlinna-Pick function) is holomorphic in the upper half plane and

$$
\operatorname{Im} f(z) \geq 0 \quad \text { whenever } \operatorname{Im} z>0 \text {. }
$$

Remark 2.5. For Jacobi operators, the inverse spectral theory is based on the fact that, from the Weyl $m$-function (or, equivalently, $\rho$ ), one uniquely recovers the matrix (2.2) and the boundary condition at infinity that defines the self-adjoint extension if necessary. This is done by means of either the discrete Riccati equation (see [13, Eq. 2.15], [37, Eq. 2.23]) or the method of orthonormalization of the polynomial sequence $\left\{t^{k}\right\}_{k=0}^{\infty}$ in $L_{2}(\mathbb{R}, \rho)$ [3, Chap. 7, Sec. 1.5]. 
If (2.2) is the matrix representation of a non-self-adjoint operator, then the condition at infinity may be found by the method exposed in [32, Sec. 2].

\section{Green functions for Jacobi operators}

We begin this section by introducing some concepts and laying out the notation.

Let $M \subset \mathbb{Z}$ and consider a sequence $\left\{M_{n}\right\}_{n=1}^{\infty}$ of finite sets containing only consecutive integers and such that

i) $M_{n} \subset M_{n+1}$ for any $n \in \mathbb{N}$,

ii) $\cup_{n=1}^{\infty} M_{n}=M$.

If, for a collection of complex numbers $\left\{r_{k}\right\}_{k \in M}$,

$$
\lim _{n \rightarrow \infty} \sum_{k \in M_{n}} r_{k}=s
$$

for any sequence $\left\{M_{n}\right\}_{n=1}^{\infty}$ satisfying i) and ii), then we define

$$
\sum_{k \in M} r_{k}:=s
$$

and say that $\sum_{k \in M} r_{k}$ converges to $s$. For any finite subset $F$ of $M$, we also consider

$$
\sum_{k \in M \backslash F} r_{k}:=\sum_{k \in M} r_{k}-\sum_{k \in F} r_{k}
$$

The expressions

$$
\prod_{k \in M} r_{k} \quad \text { and } \quad \prod_{k \in M \backslash F} r_{k}
$$

are defined analogously.

For every $z \in \mathbb{C} \backslash \rho(J)$, define

$$
\psi(z):=(J-z I)^{-1} \delta_{1}
$$

It is known that [3, Chap. 7 Eq. 1.39] that for each $z \in \mathbb{C} \backslash \rho(J)$ there exists a unique complex number $m(z)$ such that

$$
\psi(z)=m(z) \pi(z)+\xi(z) .
$$

The notation here corresponds to the fact that the number $m(z)$ is actually the value of the Weyl $m$-function at $z$. 
Definition 3.1. Given a subspace $\mathcal{G} \subset l_{2}(\mathbb{N})$, let $P_{\mathcal{G}}$ be the orthogonal projection onto $\mathcal{G}$ and $\mathcal{G}^{\perp}:=\left\{\phi \in l_{2}(\mathbb{N}):\langle\phi, \psi\rangle=0 \forall \psi \in \mathcal{G}\right\}$. Also, define the subspace $\mathcal{F}_{n}:=\operatorname{span}\left\{\delta_{k}\right\}_{k=1}^{n}$. In the Hilbert space $\mathcal{F}_{n}^{\perp}$, consider the operator

$$
J_{n}^{+}:=P_{\mathcal{F}_{n}^{\perp}} J\left\lceil_{\mathcal{F}_{n}^{\perp}}, \quad n \in \mathbb{N} \backslash\{1\} .\right.
$$

Similarly, in the space $\mathcal{F}_{n-1}$, consider

$$
J_{n}^{-}:=P_{\mathcal{F}_{n-1}} J \uparrow_{\mathcal{F}_{n-1}}, \quad n \in \mathbb{N} \backslash\{1\} .
$$

Here, we have used the notation $J \uparrow_{\mathcal{G}}$ for the restriction of $J$ to the set $\mathcal{G}$, that is, $\operatorname{dom}\left(J \curlyvee_{\mathcal{G}}\right)=\operatorname{dom}(J) \cap \mathcal{G}$. The corresponding Weyl $m$-functions of these operators are

$$
m_{n}^{+}(z):=\left\langle\delta_{n+1},\left(J_{n}^{+}-z I\right)^{-1} \delta_{n+1}\right\rangle, \quad m_{n}^{-}(z):=\left\langle\delta_{n-1},\left(J_{n}^{-}-z I\right)^{-1} \delta_{n-1}\right\rangle .
$$

The operator $J_{n}^{+}$is a self-adjoint extension of the operator whose matrix representation with respect to the basis $\left\{\delta_{k}\right\}_{k=n}^{\infty}$ of the space $\mathcal{F}_{n}^{\perp}$ is $(2.2)$ with the first $n$ rows and $n$ columns removed. When $J_{0}$ is not essentially self-adjoint, $J_{n}^{+}$has the same boundary conditions at infinity as the operator $J$. Clearly, the operator $J_{n}^{-}$lives in an $(n-1)$-dimensional space.

Definition 3.2. For any $n \in \mathbb{N}$, we use the following notation

$$
G(z, n):=\left\langle\delta_{n},(J-z I)^{-1} \delta_{n}\right\rangle, \quad z \notin \sigma(J)
$$

and call $G(z, n)$ the $n$-th Green function of the Jacobi operator $J$. Observe that $G(z, 1)=m(z)$ (See Definition 2.4).

In view of (2.8) and (2.9), one has

$$
G(z, n)=\int_{\mathbb{R}} \frac{\pi_{n}^{2}(t) d \rho(t)}{t-z}, \quad z \notin \sigma(J) .
$$

Thus, for any $n \in \mathbb{N}, G(\cdot, n)$ is a Herglotz function. This function is extended analytically to the eigenvalues of $J$ which are zeros of $\pi_{n}$ since these points are removable singularities.

On the basis of the von Neumann expansion for the resolvent (cf. [38, Chap. 6, Sec. 6.1]), one has

$$
(J-z I)^{-1} \delta_{n}=-\sum_{k=0}^{N-1} \frac{J^{k}}{z^{k+1}} \delta_{n}+\frac{J^{N}}{z^{N}}(J-z I)^{-1} \delta_{n}
$$

for any $n \in \mathbb{N}$ and $z \in \mathbb{C} \backslash \sigma(J)$. From this and the fact that (see [5, Chap. 6 
Sec. 3])

$$
\left\|(J-z I)^{-1}\right\| \leq \frac{1}{|\operatorname{Im} z|},
$$

one can obtain the following asymptotic formula

$$
G(z, n)=-\frac{1}{z}+O\left(z^{-2}\right)
$$

as $z \rightarrow \infty$ along any curve away from the spectrum.

The next assertion is proven in [13, Thm. 2.8] and [11, Prop. 2.3].

Proposition 3.3. For any $n \in \mathbb{N}$

$$
G(z, n)=\frac{-1}{b_{n}^{2} m_{n}^{+}(z)+b_{n-1}^{2} m_{n}^{-}(z)+z-q_{n}},
$$

where we define $m_{1}^{-}(z) \equiv 0$.

The case $n=1$ in (3.5) is the Riccati type equation for the Weyl $m$-function [13, Eq. 2.15], [37, Eq. 2.23].

Note that, in the case when $J_{0}$ is not essentially self-adjoint, the dependence of $G$ on the choice of the self-adjoint extension is given by the fact that $m_{n}^{+}(z)$ depends on this extension.

H 1. Hypothesis: The Jacobi operator $J$ has discrete spectrum, that is,

$$
\sigma_{e s s}(J)=\emptyset
$$

The essential spectrum $\sigma_{\text {ess }}(J)$, in this case, is the accumulation points of $\sigma(J)$. Since $\widetilde{J}_{n}$ and $J$ differ by a finite-rank perturbation, it follows from the Weyl perturbation theorem that the spectrum of $\widetilde{J}_{n}$ is also discrete. For the same reason, $J_{n}^{+}$has also discrete spectrum.

Remark 3.4. If one assumes $H 1$, then the functions $m(z)$ and $G(z, n)$ are meromorphic. A consequence of being Herglotz and meromorphic, is that these functions have real and simple zeros and poles. Moreover, zeros interlace with poles, that is between two contiguous zeros there is exactly one pole and between two contiguous poles there is only one zero [23, Chap. 7, Thm.1].

Proposition 3.5. Assume $H 1$ and let $\left\{\alpha_{k}\right\}$ be the zeros of the Green function $G(z, n)$ for any $n \in \mathbb{N}$. Then there are constants $\eta_{k} \geq 0(k \in \mathbb{N})$ such that

$$
-G(z, n)^{-1}=z-q_{n}+\sum_{k \in M} \frac{\eta_{k}}{\alpha_{k}-z},
$$

where $q_{n}$ is the $n$-th element of the main diagonal of (2.2). 
Proof. By (3.5), one has

$$
\begin{aligned}
-G(z, n)^{-1} & =z-q_{n}+b_{n}^{2} m_{n}^{+}(z)+b_{n-1}^{2} m_{n}^{-}(z) \\
& =z-q_{n}+b_{n}^{2} \sum_{k \in \widetilde{M}} \frac{\tau_{k}}{c_{k}-z}+b_{n-1}^{2} \sum_{j=1}^{n-1} \frac{\kappa_{j}}{d_{j}-z},
\end{aligned}
$$

where in the last equality we have used (2.12) and the fact that, due to Hypothesis 1, $J_{n}^{+}$has discrete spectrum. Clearly, the set of the union of the elements of $\left\{c_{k}\right\}_{k \in \widetilde{M}}$ and $\left\{d_{k}\right\}_{k=1}^{n-1}$ are the zeros of $G(z, n)$.

Remark 3.6. Since $G(z, n)$ is a Herglotz meromorphic function when $\mathrm{H} 1$ is assumed, the same is true for the function $-G(z, n)^{-1}$. Using [23, Chap. 7, Thm. 2], one writes

$$
-G(z, n)^{-1}=a z+b+\sum_{k \in M} \eta_{k}\left(\frac{1}{\alpha_{k}-z}-\frac{1}{\alpha_{k}}\right),
$$

where $a \geq 0, b$ is real and $\eta_{k} \geq 0$ for all $k \in \mathbb{N}$. Comparing this last equation with the statement of Proposition 3.5 one concludes that

$$
\sum_{k \in M} \frac{\eta_{k}}{\alpha_{k}}<+\infty .
$$

Actually, it follows from (3.5) that for an infinite set of subindices $\eta_{k}=b_{n}^{2} \tau_{k}$ (see (3.6)), therefore, as a consequence of Theorem 2.1,

$$
\sum_{k \in M} \alpha_{k}^{m} \eta_{k}<\infty, \quad \text { for all } m=0,1, \ldots
$$

since $\tau_{k}$ is jump at $\alpha_{k}$ of the spectral measure of the infinite submatrix whose Weyl $m$-function is $m^{+}$.

Proposition 3.7. Let $G(z)$ be a meromorphic function. Denote by $-\eta_{k}$ the residue of $-G(z)^{-1}$ at $\alpha_{k} . G(z)$ is the $n$-th Green function of a Jacobi operator satisfying $H 1$ for some $n \in \mathbb{N} \backslash\{1\}$ if and only if all the following conditions are satisfied

1. $G(z)$ is a Herglotz function.

2. $G(z)$ obeys the asymptotics

$$
G(z)=-\frac{1}{z}+O\left(z^{-2}\right)
$$


when $z$ tends to $\infty$ along any curve away from a strip containing the real line.

3. There exists a finite set $F$ with card $F=n$ such that all the polynomials are in $L_{2}(\mathbb{R}, \rho)$ and they are dense in this space, where

$$
\rho(t):=\sum_{\substack{\alpha_{k}<t \\ k \in \mathbb{N} \backslash F}} \eta_{k}
$$

Proof. $(\Rightarrow)$ Condition 1 follows from (3.3) and (3.4) implies Condition 2. It follows from Propositions 3.3 and 3.5 that

$$
\sum_{k \in M} \frac{\eta_{k}}{\alpha_{k}-z}=\left(\sum_{k \in M \backslash F}+\sum_{k \in F}\right) \frac{\eta_{k}}{\alpha_{k}-z},
$$

where the infinite sum in the r.h.s. of the last equation is the Weyl $m$-function of a semi-infinite Jacobi operator. Finally one recurs to Theorem 2.1 to show that Condition 3 holds.

$(\Leftarrow)$ Since $-G(z)^{-1}$ is also Herglotz, one has (3.7). Condition 3 implies in particular that

$$
\sum_{k \in M} \eta_{k}<+\infty
$$

Thus, (3.8) holds and then one can write

$$
-G(z, n)^{-1}=a z+\widetilde{b}+\sum_{k \in M} \frac{\eta_{k}}{\alpha_{k}-z} .
$$

Because of Condition 2, $a=1$. Take the set $F$ given in Condition 3 and write

$$
-G(z, n)^{-1}=z+\widetilde{b}+\left(\sum_{k \in M \backslash F}+\sum_{k \in F}\right) \frac{\eta_{k}}{\alpha_{k}-z},
$$

where, due to Theorem 2.1 and Condition 3,

$$
\sum_{k \in M \backslash F} \frac{\eta_{k}}{\alpha_{k}-z}
$$

is the Weyl $m$-function (modulo a positive constant factor) of some semi-infinite 
Jacobi operator. On the other hand,

$$
\sum_{k \in F} \frac{\eta_{k}}{\alpha_{k}-z}
$$

is the Weyl $m$-function (modulo a positive constant factor) of a finite Jacobi matrix. Finally, Proposition 3.3 completes the proof.

\section{Direct spectral analysis}

We begin this section by defining the function

$$
\widetilde{G}(z, k):=\left\langle\delta_{k},\left(\widetilde{J}_{n}-z I\right)^{-1} \delta_{k}\right\rangle
$$

to be considered alongside the function $G(z, k)$. Define

$$
\mathfrak{M}_{k}(z):=\frac{G(z, k)}{\widetilde{G}(z, k)}
$$

This function is extended to the points that are removable singularities.

The following formula plays an important role for the comparative spectral analysis of $J$ and $\widetilde{J}_{n}$ [11, Lem. 3.1].

$$
\mathfrak{M}_{k}(z)=\theta^{2}+\left(1-\theta^{2}\right)(\gamma-z) G(z, k),
$$

where

$$
\gamma:=\frac{\theta^{2} h}{1-\theta^{2}}
$$

This formula follows from (3.5).

The following assertion reproduces the one of [11, Cor. 3.2]. This result can be seen by comparing the formula (3.5) for $G(z, n)$ and $\widetilde{G}(z, n)$.

Proposition 4.1. For any $n \in \mathbb{N}$, the function $G(z, n)$ vanishes if and only if $\widetilde{G}(z, n)$ vanishes.

Proposition 4.2. Let $n \in \mathbb{N} \backslash\{1\}$. The set of common eigenvalues of $J$ and $\widetilde{J}_{n}$, different from $\gamma$, is equal to the set of common eigenvalues of $J_{n}^{+}$and $J_{n}^{-}$, different from $\gamma$. The function $G(z, n)$ vanishes at these eigenvalues.

Proof. The fact that

$$
\sigma\left(J_{n}^{+}\right) \cap \sigma\left(J_{n}^{-}\right) \subset \sigma(J) \cap \sigma\left(\widetilde{J}_{n}\right)
$$


has been proven in [11, Prop. 3.3]. To prove the converse contention, suppose that $\lambda$ is in $\sigma(J) \cap \sigma\left(\widetilde{J}_{n}\right)$. Then, by [11, Lem. 2.8], $\lambda$ is either a zero or a pole of $G(z, n)$. But [11, Lem. 3.5] implies that, if $\lambda \neq \gamma$, then $\lambda$ cannot be a pole of $G(z, n)$. The fact that $\lambda$ is a zero of $G(z, n)$ and an eigenvalue of $J$ yields through [11, Lems. 2.8 and 2.9, and Cor. 2.3] that $\lambda$ is a common eigenvalue of $J_{n}^{+}$and $J_{n}^{-}$. The last assertion is [11, Lem. 3.5].

Remark 4.3. A consequence of the previous proposition is that the number of common eigenvalues different from $\gamma$ is not greater than $n-1$. Moreover these common eigenvalues are the same for any $\theta \in(0,1)$ and $h \in \mathbb{R}$.

N 1. Notation: Assume H 1 and denote $J(\theta, h):=\widetilde{J}_{n}$ to emphasize the dependence on $\theta$ and $h$ of the operator $\widetilde{J}_{n}$ (recall that $\widetilde{J}_{n}$ is the operator whose matrix representation is given by (2.5) $)$. Denote the spectrum of $J(\theta, h)$ by $\left\{\lambda_{k}(\theta, h)\right\}_{k \in M}$. Note that this sequence has no accumulation points for any fixed $\theta>0$ and $h \in \mathbb{R}$. Let

$$
\pi(\theta, h)=\left\{\pi_{j}(\theta, h)\right\}_{j=1}^{\infty}
$$

be the eigenvector of $J(\theta, h)$ corresponding to $\lambda_{k}(\theta, h)$ normalized as before (see (2.7)), i. e.,

$$
\left\langle\delta_{1}, \pi(\theta, h)\right\rangle=1
$$

Note that the polynomial $\pi_{n}(\theta, h)$ of degree $n-1$ is evaluated at the point $\lambda_{k}(\theta, h)$.

Lemma 4.4. Assume $H 1$ and consider $N 1$. For any $k \in M, \theta>0$ and $h \in \mathbb{R}$, one has

a)

$$
\frac{d}{d \theta} \lambda_{k}(\theta, h)=\frac{2 \pi_{n}}{\|\pi\|^{2}}\left(b_{n-1} \pi_{n-1}+b_{n} \pi_{n+1}+\pi_{n} \theta\left(q_{n}+h\right)\right),
$$

where we have abbreviated $\pi:=\pi(\theta, h)$ and $\pi_{n}:=\pi_{n}(\theta, h)$.

b)

$$
\frac{d}{d h} \lambda_{k}(\theta, h)=\frac{\theta^{2} \pi_{n}^{2}(\theta, h)}{\|\pi(\theta, h)\|^{2}}
$$

Proof. Observe that if $A, A^{\prime}$ are symmetric operators with the same domain and $A f=\lambda f, A^{\prime} f^{\prime}=\lambda^{\prime} f^{\prime}$, then

$$
\left\langle f,\left(A^{\prime}-A\right) f^{\prime}\right\rangle=\left\langle f, \lambda^{\prime} f^{\prime}\right\rangle-\left\langle f, A f^{\prime}\right\rangle=\left\langle f, \lambda^{\prime} f^{\prime}\right\rangle-\left\langle A f, f^{\prime}\right\rangle=\left(\lambda^{\prime}-\lambda\right)\left\langle f, f^{\prime}\right\rangle .
$$


Proof of a)

Since $h$ will remain fixed we do not write the dependence on $h$. Pick any small real $\tau$. Since the domain of $J(\theta, h)$ does not depend on $\theta$, using (4.4), we have, similar to [11, Prop. 3.1],

$$
\left(\lambda_{k}(\theta+\tau)-\lambda_{k}(\theta)\right)\langle\pi(\theta, \pi(\theta+\tau\rangle=\langle\pi(\theta),(J(\theta+\tau)-J(\theta)) \pi(\theta+\tau)\rangle .
$$

Let us calculate the inner product of the right hand side of the above equality.

Note that

$$
J(\theta+\tau)-J(\theta)=\left(\begin{array}{ccccccc}
0 & 0 & 0 & 0 & 0 & 0 & \cdots \\
0 & \ddots & \ddots & 0 & 0 & 0 & \cdots \\
0 & \ddots & 0 & \tau b_{n-1} & 0 & 0 & \cdots \\
0 & 0 & \tau b_{n-1} & \left(2 \tau \theta+\tau^{2}\right)\left(q_{n}+h\right) & \tau b_{n} & 0 & \cdots \\
0 & 0 & 0 & \tau b_{n} & 0 & 0 & \\
0 & 0 & 0 & 0 & 0 & 0 & \ddots \\
\vdots & \vdots & \vdots & \vdots & & \ddots & \ddots
\end{array}\right)
$$

Using the notation $\pi:=\pi(\theta), \pi_{n}:=\pi_{n}(\theta), \pi^{\prime}:=\pi(\theta+\tau), \pi_{n}^{\prime}:=\pi_{n}^{\prime}(\theta+\tau)$, one obtains

$$
\begin{aligned}
& \left\langle\pi,(J(\theta+\tau)-J(\theta)) \pi^{\prime}\right\rangle= \\
& \tau\left(b_{n-1} \pi_{n-1} \pi_{n}^{\prime}+\pi_{n}\left[b_{n-1} \pi_{n-1}^{\prime}+\pi_{n}^{\prime}(2 \theta+\tau)\left(q_{n}+h\right)+b_{n} \pi_{n+1}^{\prime}\right]+\pi_{n+1} \pi_{n}^{\prime} b_{n} .\right)
\end{aligned}
$$

It follows from (4.5) that

$$
\begin{aligned}
& \frac{\lambda_{k}(\theta+\tau)-\lambda_{k}(\theta)}{\tau}= \\
& \frac{b_{n-1} \pi_{n-1} \pi_{n}^{\prime}+\pi_{n}\left[b_{n-1} \pi_{n-1}^{\prime}+\pi_{n}^{\prime}(2 \theta+\tau)\left(q_{n}+h\right)+b_{n} \pi_{n+1}^{\prime}\right]+\pi_{n+1} \pi_{n}^{\prime} b_{n}}{\left\langle\pi, \pi^{\prime}\right\rangle}
\end{aligned}
$$

Taking the limit $\tau \rightarrow 0$ and using Lemma 4.6 and Remark 4.7 we get the proof of a).

Proof of $b$ ) 
We proceed similarly. Note that

$$
J(h+\delta)-J(h)=\left(\begin{array}{ccccccc}
0 & 0 & 0 & 0 & 0 & 0 & \cdots \\
0 & \ddots & \ddots & 0 & 0 & 0 & \cdots \\
0 & \ddots & 0 & 0 & 0 & 0 & \cdots \\
0 & 0 & 0 & \theta^{2} \delta & 0 & 0 & \cdots \\
0 & 0 & 0 & 0 & 0 & 0 & \\
0 & 0 & 0 & 0 & 0 & 0 & \ddots \\
\vdots & \vdots & \vdots & \vdots & & \ddots & \ddots
\end{array}\right) .
$$

Therefore,

$$
\langle\pi(h),(J(h+\delta)-J(h)) \pi(h+\delta)\rangle=\theta^{2} \delta \pi_{n}(h) \pi_{n}(h+\delta),
$$

where since $\theta$ remains fixed, we have not written the dependence on $\theta$. Using (4.4), one has

$$
\frac{\lambda_{k}(h+\delta)-\lambda_{k}(h)}{\delta}=\frac{\theta^{2} \pi_{n}(h) \pi_{n}(h+\delta)}{\langle\pi(h), \pi(h+\delta)\rangle} .
$$

Taking the limit $\delta \rightarrow 0$ and using Lemma 4.6 and Remark 4.7, we conclude the proof of b)

The following result is well known (see [29, Last Thm. in Sec.135]).

Proposition 4.5. Let $\left\{A_{n}\right\}_{n=1}^{\infty}$ and $A$ be self-adjoint operators all having the same domain and such that

$$
\left\|A_{n}-A\right\| \underset{n \rightarrow \infty}{\longrightarrow} 0
$$

Let $E_{n}$ and $E$ be the corresponding spectral families. If I is the interval $\left(\mu_{1}, \mu_{2}\right)$ with $\mu_{1}, \mu_{2} \notin \sigma(A)$, then

$$
\left\|E_{n}(I)-E(I)\right\| \underset{n \rightarrow \infty}{\longrightarrow} 0 .
$$

We shall need the following result

Lemma 4.6. Assume $H 1$ and consider $N 1$.

a) For any fixed $h \in \mathbb{R}, \| \pi(\theta+\tau, h))-\pi(\theta, h) \| \underset{\tau \rightarrow 0}{\longrightarrow} 0$.

b) For any fixed $\theta>0, \| \pi(\theta, h+\delta))-\pi(\theta, h) \| \underset{\delta \rightarrow 0}{\longrightarrow} 0$. 
Proof. We prove a), the proof of b) is analogous. From (4.6) we see that

$$
\|J(\theta+\tau)-J(\theta)\| \underset{\tau \rightarrow 0}{\longrightarrow} 0 .
$$

Let $I$ be the interval $\left(\mu_{1}, \mu_{2}\right)$ with $\mu_{1}, \mu_{2} \notin \sigma(J(\theta))$ and such that $\sigma(J(\theta)) \cap I=$ $\{\lambda(\theta\}$. By the definition of $\pi(\theta)$, we have

$$
\pi(\theta)=k E_{\theta}(I) \delta_{1}
$$

where $k$ is a constant and $E_{\theta}$ is the spectral family of $J(\theta)$. Since $1=\left\langle k E_{\theta}(I) \delta_{1}, \delta_{1}\right\rangle$, one has

$$
k=\frac{1}{\left\|E_{\theta}(I)\right\|^{2}} .
$$

Thus, if $\tau$ is small enough, then

$$
\pi(\theta+\tau)=\frac{1}{\left\|E_{\theta+\tau}(I)\right\|^{2}} E_{\theta+\tau}(I) \delta_{1} .
$$

It follows from Proposition 4.5 that

$$
E_{\theta+\tau}(I) \underset{\tau \rightarrow 0}{\stackrel{\|\cdot\|}{\longrightarrow}} E_{\theta}(I),
$$

therefore

$$
\| \pi(\theta+\tau, h))-\pi(\theta, h) \| \underset{\tau \rightarrow 0}{\longrightarrow} 0 .
$$

Remark 4.7. Recalling that $\pi_{n}(\theta, h)$ is the polynomial of first kind evaluated at $\lambda_{k}(\theta, h)$, one has, for fixed $h$,

$$
\left|\pi_{n}(\theta, h)-\pi_{n}(\theta+\tau, h)\right| \underset{\tau \rightarrow 0}{\longrightarrow} 0 .
$$

Indeed,

$$
\begin{aligned}
\left|\pi_{n}(\theta, h)-\pi_{n}(\theta+\tau, h)\right| & =\left|\left\langle\pi(\theta, h), \delta_{n}\right\rangle-\left\langle\pi(\theta+\tau, h), \delta_{n}\right\rangle\right| \\
& =\left|\left\langle\pi(\theta, h)-\pi(\theta+\tau, h), \delta_{n}\right\rangle\right| \\
& \leq\|\pi(\theta, h)-\pi(\theta+\tau, h)\| \underset{\tau \rightarrow 0}{\longrightarrow} 0 .
\end{aligned}
$$

Analogously, for fixed $\theta$,

$$
\left|\pi_{n}(\theta, h)-\pi_{n}(\theta, h+\delta)\right| \underset{\delta \rightarrow 0}{\longrightarrow} 0 .
$$


Lemma 4.8. Assume $H 1$ and consider $N 1$. For fixed $h \in \mathbb{R}$ and $n \in \mathbb{N}$,

$$
\sum_{k \in M} \frac{\left|\lambda_{k}(\theta, h)\right|^{m}}{\alpha_{k}(\theta, h)}
$$

converges uniformly for $\theta \in\left[\theta_{1}, \theta_{2}\right]$. Moreover, for fixed $\theta>0$, it converges uniformly for $h \in\left[h_{1}, h_{2}\right]$, where

$$
\alpha_{k}(\theta, h):=\|\pi(\theta, h)\|^{2}
$$

is the normalizing constants corresponding to $\lambda_{k}(\theta, h)$.

Proof. We consider the case when $h$ is fixed. The case of $\theta$ fixed is analogous. If $m$ is even, the series (4.7) converges pointwise in $\theta$ to the momentum $s_{m}:=$ $\left\langle\delta_{1}, J^{m}(\theta) \delta_{1}\right\rangle$ which is a continuous function of $\theta$ since it is the first entry of the matrix associated to $J(\theta)^{m}$. The terms of the series (4.7) are continuous in $\theta$ as follows from Lemma 4.4 and 4.6. Therefore, the series is uniformly convergent in an interval $\left[\theta_{1}, \theta_{2}\right]$ (see [39, Sec. 1.31]. If $m$ is odd

$$
\left|\frac{\lambda_{k}(\theta, h)^{m}}{\alpha_{k}(\theta, h)}\right| \leq \frac{\lambda_{k}(\theta, h)^{m+1}}{\alpha_{k}(\theta, h)}
$$

whenever $\left|\lambda_{k}(\theta, h)\right|>1$. Hence, the series (4.7) also converges uniformly in this case for $\theta \in\left[\theta_{1}, \theta_{2}\right]$.

Theorem 4.9. Assume $H 1$, consider $N 1$, and abbreviate $\lambda_{k}:=\lambda_{k}(1,0)$ and $\mu_{k}:=\lambda_{k}(\theta, h)$. Then

$$
\sum_{n \in M}\left|\mu_{k}-\lambda_{k}\right|<\infty
$$

Proof. Let $A=[a, b]$, where $a:=\min \{\theta, 1\}, b:=\max \{\theta, 1\}$. By Lemma 4.4 a), letting $h$ fixed, we have

$$
\left|\lambda_{k}(\theta)-\lambda_{k}(1)\right| \leq \int_{A} \frac{2\left|\pi_{n}\right|}{\|\pi\|^{2}}\left(\left|b_{n-1} \pi_{n-1}\right|+\left|b_{n} \pi_{n+1}\right|+\left|\pi_{n} \theta\left(q_{n}+h\right)\right|\right) d \theta .
$$

Taking a sequence $\left\{M_{m}\right\}$ as before, we get

$$
\sum_{k \in M}\left|\lambda_{k}(\theta)-\lambda_{k}(1)\right| \leq 2 \lim _{m \rightarrow \infty} \int_{A} \sum_{k \in M_{m}} \frac{\left|\pi_{n}\right|}{\|\pi\|^{2}}\left(\left|b_{n-1} \pi_{n-1}\right|+\left|b_{n} \pi_{n+1}\right|+\left|\pi_{n} \theta\left(q_{n}+h\right)\right|\right) d \theta .
$$

Using Lemma 4.8, and recalling that $\pi_{n}$ are polynomials in $\lambda_{k}(\theta)$, we can get 
the limit inside the integral and we obtain

$$
\sum_{k \in M}\left|\lambda_{k}(\theta)-\lambda_{k}(1)\right|<\infty
$$

since the function inside the integral is continuous and $A$ is compact. Note that we have fixed $h$.

Let now $B:=[c, d]$, where $c:=\min \{0, h\}$ and $d: \max \{0, h\}$. Then, it follows from Lemma $4.4 \mathrm{~b}$ ) that

$$
\left|\lambda_{k}(1, h)-\lambda_{k}(1,0)\right|=\int_{B} \frac{\pi_{n}(1, h)^{2}}{\|\pi(1, h)\|^{2}} d h .
$$

In turn, one has

$$
\sum_{k \in M}\left|\lambda_{k}(1, h)-\lambda_{k}(1,0)\right| \leq \lim _{m \rightarrow \infty} \int_{A} \sum_{k \in M_{m}} \frac{\left|\pi_{n}(1, h)\right|}{\|\pi(1, h)\|^{2}} d h .
$$

We get the limit inside the integral since the convergence is uniform by Lemma 4.8 and recalling that $\pi_{n}$ is a polynomial evaluated at $\lambda_{k}(1, h)$. Therefore

$$
\sum_{k \in M}\left|\lambda_{k}(1, h)-\lambda_{k}(1,0)\right| \leq \int_{A} \sum_{k \in M} \frac{\left|\pi_{n}(1, h)\right|}{\|\pi(1, h)\|^{2}} d h<\infty .
$$

Now, apply the triangle inequality to obtain

$$
\sum_{k \in M}\left|\lambda_{k}(\theta, h)-\lambda_{k}(1,0)\right| \leq \sum_{k \in M}\left|\lambda_{k}(\theta, h)-\lambda_{k}(1, h)\right|+\sum_{k \in M}\left|\lambda_{k}(1, h)-\lambda_{k}(1,0)\right| .
$$

The previous result is related to [21, Thm. II] which states that the result holds for some enumeration.

Proposition 4.10. Assume $H 1$. The set of zeros of $\mathfrak{M}_{k}(z)$ coincides with $\sigma\left(\widetilde{J}_{n}\right) \backslash \sigma(J)$, while the set of poles is $\sigma(J) \backslash \sigma\left(\widetilde{J}_{n}\right)$.

Proof. It follows from [11, Cor. 3.1] that the zeros and poles of $\mathfrak{M}_{k}(z)$ are given by the poles of $\widetilde{G}(z, k)$ and $G(z, k)$ respectively. By Definition 3.2 any pole of $G(z, k)$ is an eigenvalue of $J$. If a pole of $G$ different from $\gamma$ were an eigenvalue of $\widetilde{J}_{n}$, then a contradiction follows from [11, Lem. 3.2]. Note that, as a consequence of [11, Thm. 3.3], $\gamma$ is not a pole of $\mathfrak{M}_{k}(z)$. We have established that the poles of $\mathfrak{M}_{k}(z)$ are in $\sigma(J) \backslash \sigma\left(\widetilde{J}_{n}\right)$. The converse inclusion follows directly from [11, Lem. 2.2]. By means of [11, Lem. 3.4 and Thm.3.3] one proof that set of zeros of $\mathfrak{M}_{k}(z)$ equals $\sigma\left(\widetilde{J}_{n}\right) \backslash \sigma(J)$. 
Lemma 4.11. Let $\left\{\lambda_{k}\right\}_{k \in M}$ and $\left\{\mu_{k}\right\}_{k \in M}$ be sequences such that (4.8) holds. Let $\mathcal{B}=\cup_{k \in M} B_{k}$, where $B_{k}=\left\{z \in \mathbb{C}:\left|z-\lambda_{k}\right|<a\right\}$. Then

$$
\prod_{k \in M} \frac{z-\mu_{k}}{z-\lambda_{k}}
$$

converges uniformly in $\mathbb{C} \backslash \mathcal{B}$ and

$$
\lim _{\substack{|z| \rightarrow \infty \\ z \notin \mathcal{B}}} \prod_{k \in M} \frac{z-\mu_{k}}{z-\lambda_{k}}=1
$$

Proof. Since $z \notin \mathcal{B}$,

$$
\left|\frac{\lambda_{k}-\mu_{k}}{z-\lambda_{k}}\right|<\frac{1}{a}\left|\lambda_{k}-\mu_{k}\right|
$$

for all $k \in M$. Therefore

$$
\sum_{k \in M}\left|\frac{\lambda_{k}-\mu_{k}}{z-\lambda_{k}}\right|
$$

converges uniformly in $\mathbb{C} \backslash \mathcal{B}$ [31, Thm. 7.10]. In turn, this implies

$$
\prod_{k \in M}\left(1+\frac{\lambda_{k}-\mu_{k}}{z-\lambda_{k}}\right)=\prod_{k \in M} \frac{z-\mu_{k}}{z-\lambda_{k}}
$$

converges uniformly in in $\mathbb{C} \backslash \mathcal{B}$ (see for instance [39, Sec. 1.44]).

Now, for $M_{n}$ such that $\operatorname{card}\left(M_{n}\right)=n$, one has

$$
\left|\prod_{k \in M} \frac{z-\mu_{k}}{z-\lambda_{k}}-1\right| \leq\left|\prod_{k \in M} \frac{z-\mu_{k}}{z-\lambda_{k}}-\prod_{k \in M_{n}} \frac{z-\mu_{k}}{z-\lambda_{k}}\right|+\left|\prod_{k \in M_{n}} \frac{z-\mu_{k}}{z-\lambda_{k}}-1\right| .
$$

If $n$ is sufficiently large the first term in the r. h. s. is arbitrarily small uniformly in $z \notin \mathcal{B}$. For large $|z|$, the second term is also arbitrarily small.

Proposition 4.12. Assume H1. Fix an arbitrary $n \in \mathbb{N}$. Assume that $\sigma(J) \backslash$ $\sigma\left(\widetilde{J}_{n}\right)=\left\{\lambda_{k}\right\}_{k \in M}$, where the sequence is strictly increasing. Then there is an enumeration of $\sigma\left(\widetilde{J}_{n}\right) \backslash \sigma(J)$ such that $\sigma\left(\widetilde{J}_{n}\right) \backslash \sigma(J)=\left\{\mu_{k}\right\}_{k \in M}$ and

$$
\mathfrak{M}_{n}(z)=\prod_{k \in M} \frac{z-\mu_{k}}{z-\lambda_{k}} .
$$

Proof. Assume that $J$ is not semibounded from above. Let $\lambda_{k_{0}}$ be the eigenvalue nearest to zero. Since $G(z, n)$ is a meromorphic Herglotz function, it follows 
from Proposition A.1 and Remark A.2 that

$$
G(z, n)=C \frac{\left(z-\eta_{k_{0}}\right)\left(z-\eta_{k_{0}-1}\right)}{\left(z-\lambda_{k_{0}}\right)\left(z-\lambda_{k_{0}-1}\right)} \prod_{\substack{k \in M \\ k \neq k_{0}, k_{0}-1}}\left(1-\frac{z}{\eta_{k}}\right)\left(1-\frac{z}{\lambda_{k}}\right)^{-1}
$$

where $\left\{\eta_{k}\right\}_{k \in M}$ are the zeros of $G(z, n), C>0$, and

$$
\lambda_{k}<\eta_{k}<\lambda_{k+1} \quad \forall k \in M
$$

Taking into account Proposition 4.1, one also has from Proposition A.1 and Remark A.2 that

$$
\widetilde{G}(z, n)=\widetilde{C} \frac{\left(z-\eta_{k_{0}-1}\right)\left(z-\eta_{k_{0}}\right)\left(z-\eta_{k_{0}+1}\right)}{\left(z-\mu_{k_{0}-1}\right)\left(z-\mu_{k_{0}}\right)\left(z-\mu_{k_{0}+1}\right)} \prod_{\substack{k \in M \\ k \neq k_{0}, k_{0} \pm 1}}\left(1-\frac{z}{\eta_{k}}\right)\left(1-\frac{z}{\mu_{k}}\right)^{-1}
$$

where $\widetilde{C}>0$ and

$$
\mu_{k}<\eta_{k}<\mu_{k+1} \quad \forall k \in M \text {. }
$$

Since the enumeration of the sequence $\left\{\eta_{k}\right\}_{k \in M}$ does not change, we have taken into account that $\mu_{k_{0}+1}$ could be zero.

For any values of the perturbative parameters $\theta$ and $h$, the eigenvalue $\lambda_{k}(\theta, h)$ (see the notation introduced before Lemma 4.4) is constrained between $\eta_{k-1}$ and $\eta_{k}$ which do not move as $\theta$ and $h$ change (see Proposition 4.1). Therefore the enumeration of the sequence $\left\{\mu_{k}\right\}_{k \in M}$ is such that $\lambda_{k}(\theta, h)=\mu_{k}$ for any values of the perturbative parameters.

Consider a sequence $\left\{M_{n}\right\}_{n=1}^{\infty}$ of subsets of $M$, such that $M_{n} \subset M_{n+1}$ and $\cup_{n} M_{n}=M$. One has

$$
\begin{aligned}
\mathfrak{M}_{n}(z) & =C^{\prime} \prod_{j=k_{0}-1}^{k_{0}+1} \frac{z-\mu_{j}}{z-\lambda_{j}} \lim _{n \rightarrow \infty} \frac{\prod_{\substack{k \in M_{0}, k_{0} \pm 1 \\
k \neq k_{k}}}\left(1-\frac{z}{\eta_{k}}\right)\left(1-\frac{z}{\lambda_{k}}\right)^{-1}}{\prod_{\substack{k \in M_{n} \\
k \neq k_{0}, k_{0} \pm 1}}\left(1-\frac{z}{\eta_{k}}\right)\left(1-\frac{z}{\mu_{k}}\right)^{-1}} \\
& =C^{\prime} \prod_{j=k_{0}-1}^{k_{0}+1} \frac{z-\mu_{j}}{z-\lambda_{j}} \prod_{\substack{k \in M \\
k \neq k_{0}, k_{0} \pm 1}}\left(1-\frac{z}{\mu_{k}}\right)\left(1-\frac{z}{\lambda_{k}}\right)^{-1} .
\end{aligned}
$$


By Theorem 4.9, it follows that

$$
\prod_{\substack{k \in M \\ k \neq k_{0}, k_{0} \pm 1}} \frac{\lambda_{k}}{\mu_{k}} \text { converges. }
$$

Thus, one writes

$$
\prod_{\substack{k \in M \\ k \neq k_{0}, k_{0} \pm 1}}\left(1-\frac{z}{\mu_{k}}\right)\left(1-\frac{z}{\lambda_{k}}\right)^{-1}=\prod_{\substack{k \in M \\ k \neq k_{0}, k_{0} \pm 1}} \frac{\lambda_{k}}{\mu_{k}} \prod_{\substack{k \in M \\ k \neq k_{0}, k_{0} \pm 1}} \frac{z-\mu_{k}}{z-\lambda_{k}}
$$

From (3.4) it follows that

$$
\lim _{\substack{z \rightarrow \infty \\ 0<|\arg z|<\pi}} \mathfrak{M}_{k}(z)=1
$$

On the other hand, according to Lemma 4.11 the second product of the r. h.s. tends to 1 along any curve away from the spectrum. This implies, together with (4.10), that

$$
C^{\prime}=\prod_{\substack{k \in M \\ k \neq k_{0}, k_{0} \pm 1}} \frac{\mu_{k}}{\lambda_{k}}
$$

The proposition is then proven for the case when $J$ is not semibounded from above. The case when $J$ is semibounded from above is treated analogously.

Remark 4.13. There is a simple expression for the quotient of the unperturbed and perturbed masses. Indeed, assume that $\gamma$ is not a pole of $G(z, n)$. If $\lambda$ is a common eigenvalue or $\gamma$, then (4.2) implies that $\mathfrak{M}_{n}(\lambda)=\theta^{2}$. Thus, Proposition 4.12 below and 2.6 yields

$$
\prod_{k \in M} \frac{\lambda-\mu_{k}}{\lambda-\lambda_{k}}=\frac{m_{n}}{m_{n}+\Delta m_{n}}
$$

cf. [8, Rem. 1]. It is hard to imagine a more direct relation between the eigenvalues and the perturbed mass.

\section{Inverse spectral analysis}

Definition 5.1. For any Borel set $\mathcal{A}$, put

$$
\delta_{\lambda}(\mathcal{A})= \begin{cases}1 & \text { if } \lambda \in \mathcal{A} \\ 0 & \text { otherwise }\end{cases}
$$


Lemma 5.2. Let

$$
\mu=\sum_{\lambda \in M} a_{\lambda} \delta_{\lambda}
$$

where $a_{\lambda}>0$ for any $\lambda \in M$ and $M \subset \mathbb{R}$ is a discrete infinite set. Assume that the polynomials are dense in $L_{2}(\mathbb{R}, \mu)$. Let

$$
\nu=\sum_{\lambda \in F} b_{\lambda} \delta_{\lambda}-\sum_{\lambda \in M_{0} \subset M} a_{\lambda} \delta_{\lambda}
$$

where $b_{\lambda}>0$ for any $\lambda \in F$, and $F$ and $M_{0}$ are finite sets of the same cardinality. Then the polynomials are dense in $L_{2}(\mathbb{R}, \mu+\nu)$.

Proof. Since the polynomials are dense in $L_{2}(\mathbb{R}, \mu), \mu$ is either indeterminate $N$-extremal or determinate by [1, Thm. 2.3.3 Cor. 2.3.3]. By [27, Remark, p. 231] the measure

$$
\mu-a_{\lambda_{0}} \delta_{\lambda_{0}}, \quad \lambda_{0} \in M_{0}
$$

is determinate (see also in [4] Lemma B and the comment before Lemma D). Now, by [27, Thm. 5 (d) and (e)], the measure

$$
\mu-a_{\lambda_{0}} \delta_{\lambda_{0}}+b_{\lambda} \delta_{\lambda}, \quad \lambda \in F .
$$

is either determinate or indeterminate $N$-extremal.

Remark 5.3. If the cardinality of $M_{0}$ is less than the cardinality of $F$, then the previous lemma may not be true. In fact, adding just one point mass to the measure $\mu$ may destroy the density of the polynomials. For related results see [22]. We thank C. Berg and A. Duran for making this fact clear to us and M. Sodin for pertinent remarks.

Proposition 5.4. Assume $H$ 1. If $\gamma \notin \sigma(J)$, then the sequences $\sigma(J) \backslash \sigma\left(\widetilde{J}_{n}\right)$ and $\sigma\left(\widetilde{J}_{n}\right) \backslash \sigma(J)$ together with the parameter $\gamma$ uniquely determine the function $G(z, n)$. If $\gamma \in \sigma(J)$, then the sequences $\sigma(J) \backslash \sigma\left(\widetilde{J}_{n}\right)$ and $\sigma\left(\widetilde{J}_{n}\right) \backslash \sigma(J)$ together with the parameters $\theta$ and $\gamma$ uniquely determine the function $G(z, n)$.

Proof. By Proposition 4.12, one constructs the function $\mathfrak{M}_{n}(z)$. Thus, since $\gamma \notin \sigma(J), \gamma$ is not a pole of $G(z, n)$ and, then $\mathfrak{M}_{n}(\gamma)=\theta^{2}$. With the knowledge of $\gamma$ and $\theta$, one finds $G(z, n)$ from (4.2). For the second assertion we use again Proposition 4.12 to construct $\mathfrak{M}_{n}(z)$ and then find $G(z, n)$ using (4.2).

Remark 5.5. As it will be clear later (see Theorem 5.9), although the noncommon eigenvalues are sufficient for reconstructing the $n$-Green function of the Jacobi operator, they are not sufficient for reconstructing the operator itself.

Theorem 5.6. Assume H1. Let the spectra of $J$ and $\widetilde{J}_{n}$ and the parameter $\gamma$ be given so that $\gamma$ is not in the spectrum of $J$. 
(1) If the spectra do not intersect, then the data given determine $\theta$ uniquely and there are countably many pairs Jacobi operators $J, \widetilde{J}_{n}$ with the given spectra.

(2) If the spectra intersect, then the data given determine $\theta$ uniquely and there are uncountable many pairs $J, \widetilde{J}_{n}$ with the given spectra.

Proof. (1) As in the proof of Proposition 5.4 one recovers $\theta$ and, then, the function $G(z, n)$. By Propositions 3.3 and 3.5, one has

$$
z-q_{n}+b_{n}^{2} m_{n}^{+}(z)+b_{n-1}^{2} m_{n}^{-}(z)=z-q_{n}+\sum_{k \in M} \frac{\eta_{k}}{\alpha_{k}-z} .
$$

The fact that the spectra do not intersect means that $m_{n}^{-}$and $m_{n}^{+}$do not posses common poles due to Proposition 4.2. Therefore, $\left\{\alpha_{k}\right\}_{k \in M}$ is the union of the disjoint sets $\left\{\right.$ poles of $m_{n}^{+}$\} and \{poles of $m_{n}^{-}$\}. Thus, any choice of $n-1$ terms in the series of the r.h.s of (5.1) can be made to correspond to $m_{n}^{-}$ and the infinite sum containing the remaining terms corresponds to $m_{n}^{+}$due to Lemma 5.2. Indeed, Lemma 5.2 shows that if

$$
\sum_{k \in M \backslash N} \frac{\eta_{k}}{\alpha_{k}-z}
$$

is an Weyl $m$-function, then

$$
\sum_{k \in M \backslash N^{\prime}} \frac{\eta_{k}}{\alpha_{k}-z}
$$

is an Weyl $m$-function too, for any other set $N^{\prime} \operatorname{such}$ that $\operatorname{card}(N)=\operatorname{card}\left(N^{\prime}\right)$. Hence

$$
b_{n-1}^{2} m_{n}^{-}=\sum_{k \in N} \frac{\eta_{k}}{\alpha_{k}-z}, \quad b_{n}^{2} m_{n}^{+}=\sum_{k \in M \backslash N} \frac{\eta_{k}}{\alpha_{k}-z} .
$$

In view of the fact that the null moment of the spectral measure of a Jacobi operator is 1 , that is,

$$
1=\sum_{k \in M \backslash N} b_{n}^{-2} \eta_{k}=\sum_{k \in N} b_{n-1}^{-2} \eta_{k}
$$

(cf. Theorem 2.1), one has

$$
b_{n-1}^{2}:=\sum_{k \in N} \eta_{k} \quad \text { and } \quad b_{n}^{2}:=\sum_{k \in M \backslash N} \eta_{k} .
$$

Having found $b_{n}$ and $b_{n-1}$, one finds $m_{n}^{+}$and $m_{n}^{-}$. By Remark 2.5, these functions 
determine $J_{n}^{+}$and $J_{n}^{-}$. The Jacobi operator $\mathfrak{J}$ defined by $J_{n}^{+}, J_{n}^{-}, b_{n-1}, b_{n}, q_{n}$ has the function $G(z, n)$ as its Green function. We have found as many J's with this Green function as subsets of $n-1$ elements from the countably set $M$. To complete the proof, it remains to show that the spectra of $\mathfrak{J}$ and $\widetilde{\mathfrak{J}}_{n}$ coincide with the given sequences. From the Green function $G(z, n)$ of $J$, construct $\widehat{\mathfrak{M}}_{n}$ by (4.2). This function coincides with $\mathfrak{M}_{n}$ obtained from the spectra of $J$ and $\widetilde{J}_{n}$ since the Green function is the same with $\gamma$ given and $\theta$ uniquely determined. Therefore, $\widehat{\mathfrak{M}}_{n}$ and $\mathfrak{M}_{n}$ have the same poles and zeros which are $\sigma(J)$ and $\sigma\left(\widetilde{J}_{n}\right)$, respectively.

(2) As in the previous item, the data given allow to find $\theta$ and $G(z, n)$, so one has (5.1). Let $C \subset M$ such that $k \in C$ whenever $\alpha_{k} \in \sigma(J) \cap \sigma\left(\widetilde{J}_{n}\right)$. By Remark 4.3, $\operatorname{card}(C) \leq n-1$. Pick an arbitrary $S \subset M \backslash C$ of $n-1-\operatorname{card}(C)$ elements and for each $k \in C$ choose $\beta_{k} \in(0,1)$. Thus, it follows from (5.1) that

$$
b_{n-1}^{2} m_{n}^{-}=\sum_{k \in S} \frac{\eta_{k}}{\alpha_{k}-z}+\sum_{k \in C} \frac{\beta_{k} \eta_{k}}{\alpha_{k}-z}
$$

and

$$
b_{n}^{2} m_{n}^{+}=\sum_{k \in(M \backslash C) \backslash S} \frac{\eta_{k}}{\alpha_{k}-z}+\sum_{k \in C} \frac{\left(1-\beta_{k}\right) \eta_{k}}{\alpha_{k}-z} .
$$

Using again the fact that the null moment of the spectral measure of a Jacobi operator is 1 , one has

$$
b_{n-1}^{2}:=\sum_{k \in S} \eta_{k}+\sum_{k \in C} \beta_{k} \eta_{k} \quad \text { and } \quad b_{n}^{2}:=\sum_{k \in(M \backslash C) \backslash S} \eta_{k}+\sum_{k \in C}\left(1-\beta_{k}\right) \eta_{k} .
$$

The Jacobi operator $\mathfrak{J}$ given by $J_{n}^{+}, J_{n}^{-}, b_{n-1}, b_{n}, q_{n}$ has the Green function $G(z, n)$. Note that, for any choice of the set $S$, there are as many solutions as elements in the interval $(0,1)$. To conclude the proof, observe that by construction the common eigenvalues of $J_{n}^{+}$and $J_{n}^{-}$coincide with $\sigma(J) \cap \sigma\left(\widetilde{J}_{n}\right)$. As in (1), we show that $\widehat{\mathfrak{M}}_{n}(z)=\mathfrak{M}_{n}(z)$ which implies that the noncommon eigenvalues of $J$ and $\widetilde{J}$ coincide the ones of $\mathfrak{J}$ and $\widetilde{\mathfrak{J}}_{n}$.

Theorem 5.7. Assume H1. Let the spectra of $J$ and $\widetilde{J}_{n}$ and the parameters $\theta$ and $h$ be given. If $\left(\sigma(J) \cap \sigma\left(\widetilde{J}_{n}\right) \backslash\{\gamma\}=\emptyset\right.$ and $\mathfrak{M}_{n}(\gamma)>\theta^{2}$, then there are countably many solutions of the inverse problem. In all other cases when $\gamma$ is in the spectrum of $J$, there are uncountably many solutions of the inverse problem.

Proof. If we have $\theta$ and $h$ then by (4.3) we get $\gamma$ and using Proposition 5.4 we recover $G(z, n)$. To prove there are countably many solutions we shall show that there are no common poles of $m_{n}^{-}$and $m_{n}^{+}$and the analysis will proceed 
then completely analogous to (1) of Theorem 5.6. Since the spectra of $J$ and $\widetilde{J}_{n}$ in this case only intersect at $\gamma$, this is the only possible common pole. This follows from Proposition 4.2, recalling the spectra of $J_{n}^{+}$and $J_{n}^{-}$are the poles of $m_{n}^{-}$and $m_{n}^{+}$respectively. But the condition $\mathfrak{M}_{n}(\gamma)>\theta^{2}$ together with with (4.2) imply that $\gamma$ is a pole of $G(z, n)$ and therefore $\gamma$ cannot be a pole of $m_{n}^{-}$ or $m_{n}^{+}$since $G(z, n)$ vanishes at these poles (cf. Proposition 3.3). We conclude that there are no common poles of $m_{n}^{-}$and $m_{n}^{+}$as was to be shown. In all other cases, when $\gamma \in \sigma(J) \cap \sigma\left(\widetilde{J}_{n}\right)$, the Weyl $m$-functions $m_{n}^{-}$and $m_{n}^{+}$have common eigenvalues and the analysis is analogous to (2) of Theorem 5.6. Notice that if $\gamma \in \sigma(J)$ and not a pole of $G(z, n)$, then $G(\gamma, n)=0$ and $\gamma$ is a common pole of $m_{n}^{-}$and $m_{n}^{+}$by [11, Cor. 2.3, Lems. 2.8, 2.9].

Remark 5.8. The previous theorem corresponds to [8, Thm.4] which deals with the case of finite mass-spring systems. In that setting, the analogous of case (1) of Theorem [5.6 yields a finite set of solutions. The other cases in [8, Thm. 4] can also be treated in a way similar to (1) and (2) of Theorem 5.6.

Theorem 5.9. Let $S$ and $\widetilde{S}$ be two infinite sequences without finite points of accumulation, $\gamma \in \mathbb{R}$ with $\gamma \notin S \cup \widetilde{S}$, and $n \in \mathbb{N}$. There is a matrix (2.2) such that $S=\sigma(J)$ and $\widetilde{S}=\sigma\left(\widetilde{J}_{n}\right)$, with $0<\theta<1$ and $h=\gamma\left(1 / \theta^{2}-1\right)$, if and only if the following conditions hold

(1) Between two consecutive points of $(S \backslash \widetilde{S}) \cup\{\gamma\}$ there is exactly one point of $\widetilde{S} \backslash S$. Any point of $\widetilde{S} \backslash S$ lies between two consecutive points of $(S \backslash \widetilde{S}) \cup\{\gamma\}$.

If the strictly increasing sequence $\left\{\lambda_{k}\right\}_{k \in M}$ coincides with $S \backslash \widetilde{S}$, then we enumerate the interlacing points $\left\{\mu_{k}\right\}_{k \in M}=\widetilde{S} \backslash S$ such that for $\beta \in(S \backslash$ $\widetilde{S}) \cup\{\gamma\}$,

$$
\begin{array}{llll}
\lambda_{k}<\mu_{k}<\beta & \text { if } \beta \leq \gamma & \text { and } & \left(\lambda_{k}, \beta\right) \cap(S \backslash \widetilde{S})=\emptyset, \\
\beta<\mu_{k}<\lambda_{k} & \text { if } \beta \geq \gamma & \text { and } & \left(\beta, \lambda_{k}\right) \cap(S \backslash \widetilde{S})=\emptyset .
\end{array}
$$

(2) The series

$$
\sum_{k \in M}\left|\lambda_{k}-\mu_{k}\right|
$$

is convergent.

(3) If $\lambda \in S \cap \widetilde{S}$, then $\mathfrak{N}(\lambda)=\mathfrak{N}(\gamma)$, where

$$
\mathfrak{N}(z):=\prod_{k \in M} \frac{z-\mu_{k}}{z-\lambda_{k}}
$$


(4) The function $\mathfrak{G}$ satisfies condition 3 of Proposition 3.7, where

$$
\mathfrak{G}(z):=\frac{\mathfrak{N}(z)-\mathfrak{N}(\gamma)}{(\mathfrak{N}(\gamma)-1)(z-\gamma)}
$$

Proof. We begin by proving that the conditions are necessary, i.e., given a matrix (2.2) such that $S=\sigma(J)$ and $\widetilde{S}=\sigma\left(\widetilde{J}_{n}\right)$ with $\theta<1$ and $h=\gamma\left(1 / \theta^{2}-1\right)$, then the sequences satisfy Conditions (1)-(4). Condition (1) is a consequence of [11, Thms. 3.8 and 3.10]. Condition (2) follows from Theorem 4.9. For this, we have to show that the set $\sigma\left(\widetilde{J}_{n}\right) \backslash \sigma(J)=\left\{\mu_{k}\right\}_{k \in M}$ is such that $\mu_{k}=\lambda_{k}(\theta, h)$ (as in Theorem 4.9). Indeed, for any values of the perturbative parameters $\theta$ and $h$, the eigenvalue $\lambda_{k}(\theta, h)$ (see the notation introduced before Lemma 4.4) is constrained between $\eta_{k-1}$ and $\eta_{k}$ which do not move as $\theta$ and $h$ change (see Proposition 4.11). Therefore the enumeration of the sequence $\left\{\mu_{k}\right\}_{k \in M}$ is such that $\lambda_{k}(\theta, h)=\mu_{k}$ for any values of the perturbative parameters.

For proving Condition (3), observe that (4.2) and Proposition 4.12 give

$$
\mathfrak{N}(z)=\prod_{k \in M} \frac{z-\mu_{k}}{z-\lambda_{k}}=\theta^{2}+\left(1-\theta^{2}\right)(\gamma-z) G(z, n)
$$

Now, [11, Lem. 3.5] implies that $\mathfrak{N}(\lambda)=\theta^{2}$ for any $\lambda \in S \cap \widetilde{S}$. Also, from (4.2), it follows that $\mathfrak{N}(\gamma)=\theta^{2}$ since $\gamma \notin S$ means that $\gamma$ is not a pole of $G(z, n)$. Condition (4) follows from Proposition 4.12, (4.2) and Proposition 3.7.

Let us now prove that the conditions are sufficient. First observe that Condition (1) implies that

$$
0<\frac{\gamma-\mu_{k}}{\gamma-\lambda_{k}}<1
$$

for any $k \in M$. Thus,

$$
0<\mathfrak{N}(\gamma)<1
$$

From (5.2), one has

$$
-\frac{\mathfrak{N}(z)}{z-\gamma}=-\lim _{n \rightarrow \infty}(z-\gamma)^{-1} \prod_{k \in M_{n}} \frac{z-\mu_{k}}{z-\lambda_{k}} .
$$

Expanding in partial fractions

$$
-\prod_{k \in M_{n}} \frac{z-\mu_{k}}{z-\nu_{k}} \frac{1}{z-\gamma}=\sum_{k \in M_{n}} \frac{\alpha_{k}}{\lambda_{k}-z}+\frac{\alpha}{\gamma-z} .
$$

Since the sequences $\left\{\mu_{k}\right\}_{k \in M}$ and $\left\{\lambda_{k}\right\}_{k \in M} \cup\{\gamma\}$ interlace according to Condition (1), we have that $\alpha_{k}>0$ for any $k \in M_{n}$ and $\alpha>0$ and, therefore, the l.h.s. 
of (5.4) is Herglotz (cf. the proof of [13, Cor. 2.5]) for any $n \in \mathbb{N}$. By definition, the l.h.s. of (5.3) is analytic outside the real axis and it is the limit of Herglotz functions, thus it is a Herglotz function. By [23, Thm. 2, Chap. 7], one has

$$
-\frac{\mathfrak{N}(z)}{z-\gamma}=a z+b+\sum_{\substack{k \in \mathcal{M} \\ k \neq 0}} A_{k}\left(\frac{1}{\nu_{k}-z}-\frac{1}{\nu_{k}}\right)+\frac{A_{0}}{\nu_{0}-z}
$$

where $a \geq 0, b \in \mathbb{R}$ and $A_{k} \geq 0$ for all $k \in \mathcal{M}$. Here only $\nu_{0}$ is allowed to be zero. Assume, without loss of generality, that $\gamma=\nu_{k_{0}}$ and $k_{0} \neq 0$. Then

$$
-\frac{\mathfrak{N}(z)}{z-\gamma}=a z+b+\sum_{\substack{k \in \mathcal{M} \\ k \neq 0, k_{0}}} A_{k}\left(\frac{1}{\nu_{k}-z}-\frac{1}{\nu_{k}}\right)+\frac{A_{0}}{\nu_{0}-z}+A_{k_{0}}\left(\frac{1}{\gamma-z}-\frac{1}{\gamma}\right) .
$$

Note that

$$
\operatorname{Res}_{z=\gamma} \frac{\mathfrak{N}(z)}{z-\gamma}:=\lim _{z \rightarrow \gamma}(z-\gamma) \frac{\mathfrak{N}(z)}{z-\gamma}=\mathfrak{N}(\gamma) .
$$

Since the residue of the r.h.s. at $\gamma=\nu_{k_{0}}$ of (5.5) is $-A_{k_{0}}$ (see the proof of [23, Chap. 7, Thm. 2]), one has

$$
-\frac{\mathfrak{N}(z)}{z-\gamma}+\frac{\mathfrak{N}(\gamma)}{z-\gamma}=a z+b+\sum_{\substack{k \in \mathcal{M} \\ k \neq 0, k_{0}}} A_{k}\left(\frac{1}{\nu_{k}-z}-\frac{1}{\nu_{k}}\right)+\frac{A_{0}}{\nu_{0}-z}-A_{k_{0}} \frac{1}{\gamma} .
$$

The last equation implies that

$$
-\frac{\mathfrak{N}(z)}{z-\gamma}+\frac{\mathfrak{N}(\gamma)}{z-\gamma}
$$

is a Herglotz function. This, in turn, yields that $\mathfrak{G}$ is Herglotz since $0<\mathfrak{N}(\gamma)<$ 1 .

Note that, as was shown in Lemma 4.11, Condition (2) implies that

$$
1=\lim _{\substack{|z| \rightarrow \infty \\ z \notin \mathcal{B}}} \prod_{k \in M} \frac{z-\mu_{k}}{z-\lambda_{k}}=\lim _{\substack{|z| \rightarrow \infty \\ z \notin \mathcal{B}}} \mathfrak{N}(z)
$$

Which in turn yields

$$
\mathfrak{G}(z)=-\frac{1}{z}+O\left(z^{-2}\right)
$$

as $z \rightarrow \infty$ along any curve away of a strip containing the real axis. Taking into account Condition (4), it follows from Proposition 3.7, that $\mathfrak{G}$ is the $n$-th Green function of a family of Jacobi operators. There is an element $J$ of this family such that $\sigma(J)=S$. Indeed, by (3.5) , there are $q_{n} \in \mathbb{R}, b_{n}, b_{n-1}>0$, and Weyl 
$m$-functions $m_{n}^{ \pm}$, such that

$$
-\mathfrak{G}(z)^{-1}=z-q_{n}+b_{n}^{2} m_{n}^{+}+b_{n-1}^{2} m_{n}^{-}
$$

Now for the spectrum of $J$ to be $S$, one can always choose the Weyl $m$-functions of the submatrices $J_{n}^{ \pm}$in such a way that $m_{n}^{ \pm}$have common poles at $S \cap \widetilde{S}$. Here Condition (3) guarantees that $\mathfrak{G}$ has zeros at $S \cap \widetilde{S}$.

Having fixed the operator $J$ such that $\sigma(J)=S$, one defines

$$
\theta:=+\sqrt{\mathfrak{N}(\gamma)} \quad \text { and } \quad h:=\gamma\left(1 / \theta^{2}-1\right)
$$

Note that $\theta<1$. Consider the Jacobi operator $J$ and the operator $\widetilde{J}_{n}$ given in (2.4). The $n$-th Green function of $J$ satisfies

$$
G(z, n)=\frac{\prod_{k \in M} \frac{z-\widetilde{\mu}_{k}}{z-\lambda_{k}}-\theta^{2}}{\left(\theta^{2}-1\right)(z-\gamma)}
$$

where $\left\{\widetilde{\mu}_{k}\right\}_{k \in M}=\sigma\left(\widetilde{J}_{n}\right)$ (see (4.2). From the definition of $\mathfrak{G}$ and our definition of $\theta$ (see (5.7)), one has

$$
\prod_{k \in M} \frac{z-\widetilde{\mu}_{k}}{z-\lambda_{k}}=\prod_{k \in M} \frac{z-\mu_{k}}{z-\lambda_{k}}
$$

for any $z \in \mathbb{C} \backslash \mathbb{R}$. The last equality implies that

$$
\mu_{k}=\widetilde{\mu}_{k} \quad \text { for any } \quad k \in M \text {. }
$$

Theorem 5.10. Let $S$ and $\widetilde{S}$ be two infinite sequences without finite points of accumulation, $\gamma \in S \cap \widetilde{S}$, and $n \in \mathbb{N}$. There is a matrix (2.2) such that $S=\sigma(J)$ and $\widetilde{S}=\sigma\left(\widetilde{J}_{n}\right)$, with $0<\theta<1$ and $h=\gamma\left(1 / \theta^{2}-1\right)$, if and only if the following conditions hold

(1) Between two consecutive points of $(S \backslash \widetilde{S}) \cup\{\gamma\}$ there is exactly one point of $\widetilde{S} \backslash S$. Any point of $\widetilde{S} \backslash S$ lies between two consecutive points of $(S \backslash \widetilde{S}) \cup\{\gamma\}$. If the strictly increasing sequence $\left\{\lambda_{k}\right\}_{k \in M}$ coincides with $S \backslash \widetilde{S}$, then we enumerate the interlacing points $\left\{\mu_{k}\right\}_{k \in M}=\widetilde{S} \backslash S$ such that for $\beta \in(S \backslash$ $\widetilde{S}) \cup\{\gamma\}$

$$
\begin{array}{llll}
\lambda_{k}<\mu_{k}<\beta & \text { if } \beta \leq \gamma & \text { and } & \left(\lambda_{k}, \beta\right) \cap(S \backslash \widetilde{S})=\emptyset \\
\beta<\mu_{k}<\lambda_{k} & \text { if } \beta \geq \gamma & \text { and } & \left(\beta, \lambda_{k}\right) \cap(S \backslash \widetilde{S})=\emptyset .
\end{array}
$$


(2) The series

$$
\sum_{k \in M}\left|\lambda_{k}-\mu_{k}\right|
$$

is convergent.

(3) If $\lambda_{1}, \lambda_{2}$ are in $(S \cap \widetilde{S}) \backslash\{\gamma\}$, then $\mathfrak{N}\left(\lambda_{1}\right)=\mathfrak{N}\left(\lambda_{2}\right) \leq \mathfrak{N}(\gamma)$, where

$$
\mathfrak{N}(z):=\prod_{k \in M} \frac{z-\mu_{k}}{z-\lambda_{k}}
$$

(4) The function

$$
\mathfrak{G}(z):= \begin{cases}\frac{\mathfrak{N}(z)-\mathfrak{N}(\omega)}{(\mathfrak{N}(\omega)-1)(z-\gamma)} & \text { if }(S \cap \widetilde{S}) \backslash\{\gamma\} \neq \emptyset \\ \frac{\mathfrak{N}(z)-\vartheta^{2}}{\left(\vartheta^{2}-1\right)(z-\gamma)} & \text { if }(S \cap \widetilde{S}) \backslash\{\gamma\}=\emptyset\end{cases}
$$

where

$$
\omega \in(S \cap \widetilde{S}) \backslash\{\gamma\}, \quad \vartheta^{2} \in \begin{cases}(0, \mathfrak{N}(\gamma)) & \text { if } \mathfrak{N}^{\prime}(\gamma) \neq 0 \\ (0, \mathfrak{N}(\gamma)] & \text { if } \mathfrak{N}^{\prime}(\gamma)=0\end{cases}
$$

satisfies condition 3 of Proposition 3.7.

Proof. The fact that (1) and (2) are necessary is proven as in Theorem 5.9. Let us prove that (3) is necessary. Note that if $S=\sigma(J)$ and $\widetilde{S}=\sigma\left(\widetilde{J}_{n}\right)$, then $\mathfrak{N}(z)=\mathfrak{M}_{n}(z)$. By [11, Lem. 3.5], $G\left(\lambda_{1}, n\right)=G\left(\lambda_{2}, n\right)=0$ for any $\lambda_{1}, \lambda_{2} \in(S \cap \widetilde{S}) \backslash\{\gamma\}$. Thus, (4.2) implies that $\mathfrak{M}_{n}\left(\lambda_{1}\right)=\mathfrak{M}_{n}\left(\lambda_{2}\right)=\theta^{2}$. Now, it follows from [11, Lem. 2.8], that $\gamma$ is either a zero or a pole of $G(z, n)$, therefore by (3.3)

$$
\operatorname{Res}_{z=\gamma} G(z, n)=-\pi_{n}^{2}(\gamma) \rho\{\gamma\} \leq 0 .
$$

Thus, using (4.2), one has

$$
\mathfrak{M}_{n}(\gamma)=\theta^{2}-\left(1-\theta^{2}\right) \operatorname{Res}_{z=\gamma} G(z, n) \geq \theta^{2} .
$$

Condition (4) follows from Proposition 3.7 and the fact that, due to Condition (3), the function $\mathfrak{G}(z)$ coincides with $G(z, n)$.

We now prove that the conditions are sufficient. First note that Condition (1) implies that

$$
0<\mathfrak{N}(\gamma)<1
$$

As in the proof of Theorem 5.9, one shows that

$$
-\frac{\mathfrak{N}(z)}{z-\gamma}
$$


is a Herglotz function. Thus, assuming without loss of generality, that $\gamma=\nu_{k_{0}}$ and $k_{0} \neq 0$. Then

$$
-\frac{\mathfrak{N}(z)}{z-\gamma}=a z+b+\sum_{\substack{k \in \mathcal{M} \\ k \neq 0, k_{0}}} A_{k}\left(\frac{1}{\nu_{k}-z}-\frac{1}{\nu_{k}}\right)+\frac{A_{0}}{\nu_{0}-z}+\mathfrak{N}(\gamma)\left(\frac{1}{\gamma-z}-\frac{1}{\gamma}\right)
$$

where we have used 5.6. Hence

$$
\begin{aligned}
& -\frac{\mathfrak{N}(z)}{z-\gamma}+\frac{\vartheta^{2}}{z-\gamma}= \\
& =a z+b+\sum_{\substack{k \in \mathcal{M} \\
k \neq 0, k_{0}}} A_{k}\left(\frac{1}{\nu_{k}-z}-\frac{1}{\nu_{k}}\right)+\frac{A_{0}}{\nu_{0}-z}+\left(\mathfrak{N}(\gamma)-\vartheta^{2}\right)\left(\frac{1}{\gamma-z}-\frac{1}{\gamma}\right) .
\end{aligned}
$$

In the case $(S \cap \widetilde{S}) \backslash\{\gamma\}=\emptyset$, since $\theta^{2} \leq \mathfrak{N}(\gamma)$, as required in Condition (4), the l. h. s. of (5.9) is a Herglotz function. When $(S \cap \widetilde{S}) \backslash\{\gamma\} \neq \emptyset$, one analogously obtains that

$$
-\frac{\mathfrak{N}(z)}{z-\gamma}+\frac{\mathfrak{N}(\omega)}{z-\gamma}
$$

is a Herglotz function due to Condition (3). By (5.8), taking into account Conditions (3) and (4), one concludes that $\mathfrak{G}$ is a Herglotz function. The rest of the proof is the same as the part of the proof of Theorem 5.9 after it is established that $\mathfrak{G}$ is a Herglotz function.

Remark 5.11. When the peturbation parameter $\theta$ is greater than 1 , one can prove results along the same lines as Theorems 5.9] and 5.10. In this case the point $\gamma$ acts as a "repeller" instead of being an "attractor".

Open problems. We have just scratched the surface of some inverse spectral theorems for Jacobi operators and many questions remain open. In the model studied here, we would like to know how many perturbations are needed to recover the system uniquely. If the perturbation takes place in the first mass, then just the spectral information provided by two spectra is enough. How many spectra do we need if the perturbation happens at the $n$-th mass? How do we determine from the spectral information where the perturbation took place? How about reconstruction results when we have partial information of the spectra or when Hypothesis 1 above does not hold?

\section{Acknowledgments}

We thank C. Berg, A. Durán, F. Marcellan and M. Sodin for valuable remarks, and R. del Rio A. for a hint to the literature. 


\section{Appendix}

For reader's convenience we give the proof of the following assertion which follows from a result due to M. G. Krein [23, Chap. 7, Thm. 1] (cf. [32, Sec. 4]).

Proposition A.1. Let $m(z)$ be the Weyl m-function of a Jacobi operator with discrete spectrum. Then

i) the zeros and poles of $m(z)$ are real, simple and interlace,

ii) the zeros $\left\{\eta_{k}\right\}_{k \in M}$ and poles $\left\{\lambda_{k}\right\}_{k \in M}$ of $m(z)$ can be enumerated in such a way that if $0 \in\left\{\eta_{k}\right\}_{k \in M} \cup\left\{\lambda_{k}\right\}_{k \in M}$, then either $\eta_{k_{0}}=0$ or $\lambda_{k_{0}}=0$ and

$$
m(z)=C \frac{z-\eta_{k_{0}}}{z-\lambda_{k_{0}}} \prod_{\substack{k \in M \\ k \neq k_{0}}}\left(1-\frac{z}{\eta_{k}}\right)\left(1-\frac{z}{\lambda_{k}}\right)^{-1},
$$

where $C<0$ and

$$
\eta_{k}<\lambda_{k}<\eta_{k+1} \quad \forall k \in M
$$

if $\sigma(J)$ is semi-bounded from above, while, $C>0$ and

$$
\lambda_{k}<\eta_{k}<\lambda_{k+1} \quad \forall k \in M
$$

otherwise.

Proof. Item i) follows from the fact that $m(z)$ is a (nonconstant) Herglotz meromorphic function and the argument principle (see the proof of [23, Chap. 7, Thm. 1]). For proving ii) first we show that the infinite product in (A.10) converges uniformly on compacts not containing the poles. Note that, due to the interlacing property, one has

$$
0<\sum_{k \in M \backslash\left\{k_{0}\right\}}\left(\frac{1}{\eta_{k}}-\frac{1}{\lambda_{k}}\right)<\sum_{k \in M \backslash\left\{k_{0}\right\}}\left(\frac{1}{\eta_{k}}-\frac{1}{\eta_{k+1}}\right)
$$

for the (A.11) case and

$$
0<\sum_{k \in M \backslash\left\{k_{0}\right\}}\left(\frac{1}{\lambda_{k}}-\frac{1}{\eta_{k}}\right)<\sum_{k \in M \backslash\left\{k_{0}\right\}}\left(\frac{1}{\lambda_{k}}-\frac{1}{\lambda_{k+1}}\right)
$$

for the A.12 case. These inequalities imply that the series

$$
\sum_{k \in M \backslash\left\{k_{0}\right\}}\left(\frac{1}{\lambda_{k}}-\frac{1}{\eta_{k}}\right)
$$


converges in both (A.11) and A.12 cases. Now, since

$$
\sum_{k \in M \backslash\left\{k_{0}\right\}}\left[\left(1-\frac{z}{\eta_{k}}\right)\left(1-\frac{z}{\lambda_{k}}\right)^{-1}-1\right]<z \sum_{k \in M \backslash\left\{k_{0}\right\}}\left(\frac{1}{\lambda_{k}}-\frac{1}{\eta_{k}}\right)\left(1-\frac{z}{\lambda_{k}}\right)^{-1}
$$

the infinite product in (A.10) converges uniformly on compacts not containing $\lambda_{k}$ for any $k \in M \backslash\left\{k_{0}\right\}$. As in the proof of [23, Chap. 7, Thm.1], one can show that when $\mathrm{A} .12$ holds, the function

$$
\frac{z-\eta_{k_{0}}}{z-\lambda_{k_{0}}} \prod_{\substack{k \in M \\ k \neq k_{0}}}\left(1-\frac{z}{\eta_{k}}\right)\left(1-\frac{z}{\lambda_{k}}\right)^{-1}
$$

is Herglotz due to

$$
0<\arg \left(\frac{1-\frac{z}{\eta_{k}}}{1-\frac{z}{\lambda_{k}}}\right)=\measuredangle \lambda_{k} z \eta_{k}<\pi
$$

and

$$
0<\sum_{k \in M} \measuredangle \lambda_{k} z \eta_{k} \leq \pi
$$

Analogously, if (A.11) takes place,

$$
-\frac{z-\eta_{k_{0}}}{z-\lambda_{k_{0}}} \prod_{\substack{k \in M \\ k \neq k_{0}}}\left(1-\frac{z}{\eta_{k}}\right)\left(1-\frac{z}{\lambda_{k}}\right)^{-1}
$$

is Herglotz since now

$$
0>\measuredangle \lambda_{k} z \eta_{k}>-\pi \text {. }
$$

Following the same reasoning as in the proof of [23, Chap. 7, Thm. 1], one shows that

$$
\frac{m(z)}{ \pm \frac{z-\eta_{k_{0}}}{z-\lambda_{k_{0}}} \prod_{\substack{k \in M \\ k \neq k_{0}}}\left(1-\frac{z}{\eta_{k}}\right)\left(1-\frac{z}{\lambda_{k}}\right)^{-1}}
$$

is a constant. For finishing the proof it remains to show that A.12 occurs when $J$ is not semibounded from above and (A.11) happens when $J$ is semibounded from above. If $J$ is semibounded from below, $J_{1}^{+}$also is. Moreover $J \leq J_{1}^{+}$, so the $\min \sigma(J) \leq \min \sigma\left(J_{1}^{+}\right)$. Since the poles of $m(z)$ constitute the spectrum of $J$ and the set of zeros is $\sigma\left(J_{1}^{+}\right)$one has that the smallest pole is less than the smallest zero, i. e. A.12. If $J$ is semibounded from above, one has $J_{1}^{+}<J$ and, then, the biggest pole is greater than the biggest zero, that is (A.11).

Remark A.2. From the proof of the previous theorem, it follows that there is 
a positive constant $C$ such that

$$
m(z)=C \prod_{j=k_{0}-n}^{k=k_{0}+n} \frac{z-\eta_{j}}{z-\lambda_{j}} \prod_{k \in M \backslash\left\{k_{0}-n, \ldots, k_{0}+n\right\}}\left(1-\frac{z}{\eta_{k}}\right)\left(1-\frac{z}{\lambda_{k}}\right)^{-1}
$$

for any $n \in \mathbb{N}$. This is used in the proof of Proposition 4.12.

\section{References}

[1] N. I. Akhiezer. The classical moment problem and some related questions in analysis. Translated by N. Kemmer. Hafner Publishing Co., New York, 1965.

[2] N. I. Akhiezer and I. M. Glazman. Theory of linear operators in Hilbert space. Dover Publications Inc., New York, 1993. Translated from the Russian and with a preface by Merlynd Nestell, Reprint of the 1961 and 1963 translations, Two volumes bound as one.

[3] J. M. Berezans'kǐr. Expansions in eigenfunctions of selfadjoint operators. Translated from the Russian by R. Bolstein, J. M. Danskin, J. Rovnyak and L. Shulman. Translations of Mathematical Monographs, Vol. 17. American Mathematical Society, Providence, R.I., 1968.

[4] C. Berg and A. J. Duran. The index of determinacy for measures and the $l^{2}$ norm of orthonormal polynomials. Trans. Amer. Math. Soc., 347(8):27952811, 1995.

[5] M. S. Birman and M. Z. Solomjak. Spectral theory of selfadjoint operators in Hilbert space. Mathematics and its Applications (Soviet Series). D. Reidel Publishing Co., Dordrecht, 1987. Translated from the 1980 Russian original by S. Khrushchëv and V. Peller.

[6] M. T. Chu and G. H. Golub. Inverse eigenvalue problems: theory, algorithms, and applications. Numerical Mathematics and Scientific Computation. Oxford University Press, New York, 2005.

[7] C. de Boor and G. H. Golub. The numerically stable reconstruction of a Jacobi matrix from spectral data. Linear Algebra Appl., 21(3):245-260, 1978.

[8] R. del Rio and M. Kudryavtsev. Inverse problems for Jacobi operators: I. Interior mass-spring perturbations in finite systems. Inverse Problems, 28(5):055007, 18, 2012. 
[9] R. del Rio, M. Kudryavtsev, and L. O. Silva. Inverse problems for Jacobi operators III: Mass-spring perturbations of semi-infinite systems. Inverse Probl. Imaging, 6(4):599-621, 2012.

[10] R. del Rio, M. Kudryavtsev, and L. O. Silva. Inverse problems for Jacobi operators II: Mass perturbations of semi-infinite mass-spring systems. $Z \mathrm{~h}$. Mat. Fiz. Anal. Geom., 9(2):165-190, 277, 281, 2013.

[11] R. del Rio and L. O. Silva. Spectral analysis for linear semi-infinite massspring systems. Math. Nachr., 288(11-12):1241-1253, 2015.

[12] M. G. Gasymov and G. S. Guselnov. On inverse problems of spectral analysis for infinite Jacobi matrices in the limit-circle case. Dokl. Akad. Nauk SSSR, 309(6):1293-1296, 1989.

[13] F. Gesztesy and B. Simon. $m$-functions and inverse spectral analysis for finite and semi-infinite Jacobi matrices. J. Anal. Math., 73:267-297, 1997.

[14] G. M. L. Gladwell. Inverse problems in vibration, volume 119 of Solid Mechanics and its Applications. Kluwer Academic Publishers, Dordrecht, second edition, 2004.

[15] L. J. Gray and D. G. Wilson. Construction of a Jacobi matrix from spectral data. Linear Algebra and Appl., 14(2):131-134, 1976.

[16] G. Š. Gusernov. The determination of the infinite Jacobi matrix from two spectra. Mat. Zametki, 23(5):709-720, 1978.

[17] R. Z. Halilova. An inverse problem. Izv. Akad. Nauk Azerbaŭdžan. SSR Ser. Fiz.-Tehn. Mat. Nauk, 1967(3-4):169-175, 1967.

[18] H. Hochstadt. On some inverse problems in matrix theory. Arch. Math. (Basel), 18:201-207, 1967.

[19] H. Hochstadt. On the construction of a Jacobi matrix from spectral data. Linear Algebra and Appl., 8:435-446, 1974.

[20] H. Hochstadt. On the construction of a Jacobi matrix from mixed given data. Linear Algebra Appl., 28:113-115, 1979.

[21] T. Kato. Variation of discrete spectra. Comm. Math. Phys., 111(3):501504, 1987.

[22] M. Langer and H. Woracek. Stability of N-extremal measures. Methods Funct. Anal. Topology, 21(1):69-75, 2015. 
[23] B. J. Levin. Distribution of zeros of entire functions, volume 5 of Translations of Mathematical Monographs. American Mathematical Society, Providence, R.I., revised edition, 1980. Translated from the Russian by R. P. Boas, J. M. Danskin, F. M. Goodspeed, J. Korevaar, A. L. Shields and H. P. Thielman.

[24] V. A. Marchenko. Introduction to the theory of inverse problems of spectral analysis. Universitetski Lekcii. Akta, Kharkov, 2005. In Russian.

[25] P. Nylen and F. Uhlig. Inverse eigenvalue problem: existence of special spring-mass systems. Inverse Problems, 13(4):1071-1081, 1997.

[26] P. Nylen and F. Uhlig. Inverse eigenvalue problems associated with springmass systems. In Proceedings of the Fifth Conference of the International Linear Algebra Society (Atlanta, GA, 1995), volume 254, pages 409-425, 1997.

[27] M. Pérez Riera and J. L. Varona Malumbres. On completeness of orthogonal systems and Dirac deltas. J. Comput. Appl. Math., 58(2):225-231, 1995.

[28] Y. M. Ram. Inverse eigenvalue problem for a modified vibrating system. SIAM J. Appl. Math., 53(6):1762-1775, 1993.

[29] F. Riesz and B. Sz.-Nagy. Functional analysis. Dover Books on Advanced Mathematics. Dover Publications, Inc., New York, 1990. Translated from the second French edition by Leo F. Boron, Reprint of the 1955 original.

[30] M. Rosenblum and J. Rovnyak. Topics in Hardy classes and univalent functions. Birkhäuser Advanced Texts: Basler Lehrbücher. [Birkhäuser Advanced Texts: Basel Textbooks]. Birkhäuser Verlag, Basel, 1994.

[31] W. Rudin. Principles of mathematical analysis. McGraw-Hill Book Co., New York-Auckland-Düsseldorf, third edition, 1976. International Series in Pure and Applied Mathematics.

[32] L. O. Silva and R. Weder. On the two spectra inverse problem for semiinfinite Jacobi matrices. Math. Phys. Anal. Geom., 9(3):263-290 (2007), 2006.

[33] L. O. Silva and R. Weder. The two-spectra inverse problem for semiinfinite Jacobi matrices in the limit-circle case. Math. Phys. Anal. Geom., 11(2):131-154, 2008.

[34] B. Smith. Infrared Spectral Interpretation: A Systematic Approach. Taylor \& Francis, 1998. 
[35] M. Spletzer, A. Raman, H. Sumali, and J. P. Sullivan. Highly sensitive mass detection and identification using vibration localization in coupled microcantilever arrays. Appl. Phys. Lett., 92:114102, 2008.

[36] M. Spletzer, A. Raman, A. Q. Wu, and X. Xu. Ultrasensitive mass sensing using mode localization in coupled microcantilevers. Appl. Phys. Lett., $88: 254102,2006$.

[37] G. Teschl. Trace formulas and inverse spectral theory for Jacobi operators. Comm. Math. Phys., 196(1):175-202, 1998.

[38] G. Teschl. Jacobi operators and completely integrable nonlinear lattices, volume 72 of Mathematical Surveys and Monographs. American Mathematical Society, Providence, RI, 2000.

[39] E. C. Titchmarsh. The theory of functions. Oxford University Press, Oxford, 1958. Reprint of the second (1939) edition. 